\title{
Divers aspects du comportement mécanique des marnes en laboratoire
}

\author{
Jean-François Serratrice ${ }^{\star}$ \\ Cerema méditerranée, 30 rue Albert-Einstein, CS 70499, 13593 Aix-en-Provence, France
}

\begin{abstract}
Résumé - Diverses illustrations du comportement mécanique des marnes sont présentées sur la base d'exemples de résultats d'essais de laboratoire. Les marnes ont été testées dans leur état naturel au cours de programmes expérimentaux réalisés dans le cadre des reconnaissances géotechniques de massifs marneux pour des projets d'ouvrages en France. Les illustrations s'adressent à la grande variété des marnes par niveaux de difficultés expérimentales croissantes, en allant des marnes homogènes tendres vers des terrains fissurés ou altérés. Les premiers exemples concernent des marnes du point de vue de leur déformabilité, leur résistance et leur caractère isotrope ou anisotrope. Des exemples sont consacrés ensuite au comportement de marnes plus ou moins indurées, puis des marnes fissurées et des marnes altérées. Les programmes expérimentaux reposent essentiellement sur des essais triaxiaux qui sont réalisés dans une gamme étendue de pressions $(0-10 \mathrm{MPa})$, ce qui permet de mettre en relief les différents aspects des comportements observés.
\end{abstract}

Mots clés : marne / comportement / haute pression / état limite / anisotropie

Abstract - Various aspects of the mechanical behavior of marls in the laboratory. Various illustrations of the mechanical behavior of marls are presented based on examples of laboratory test results. Marls were tested in their natural state over experimental programs carried out as part of the geotechnical investigations of marly subsoils for works projects in France. The illustrations are addressed to a wide variety of marls by increasing levels of experimental difficulties, going from homogeneous soft marl to fissured or weathered grounds. The first examples relate to marls deformability, their strength and their isotropic or anisotropic character. Others examples are then devoted to the behavior of more or less indurated marls, fissured marls and weathered marls. Experimental programs are mainly based on triaxial tests which are carried out in a wide pressure range $(0-10 \mathrm{MPa})$, which allows to highlight different aspects of observed behaviors.

Keywords: marl / behaviour / high pressure / limit state / anisotropy

\section{Introduction}

Les marnes constituent un groupe particulier parmi les sols indurés et les roches tendres. Sous la forme d'une grande variété lithologique de terrains argilo-carbonatés, elles occupent de nombreux étages de l'échelle stratigraphique. Mais leurs états de compacité, d'altération, structuraux et mécaniques sont divers et sont parfois à l'origine de grandes difficultés en matière d'instabilité de versants naturels et aussi dans les excavations pour les ouvrages de génie civil (déblais, puits, galeries, tunnels). Cette variété de natures et d'états confère à ces terrains un large spectre de comportements mécaniques.

Les marnes se rencontrent dans de nombreux pays dans le monde parmi lesquels figurent la Grèce (Anagnostopoulos

\footnotetext{
$\bar{\star}$ Auteur de correspondance :

jean-francois.serratrice@cerema.fr
}

et al., 1991; Kavvadas et al., 1993), l'Italie (Jamiolkowski et al., 1995; Burland et al., 1996), la France (Gaudin, 1972; Gaudin et al., 1981; Tillard-Ngan et al., 1993; Pejon et al., 1997; Gaudin et Serratrice, 1998; Maquaire et al., 2003; Raynaud et al., 2008), l'Espagne (Serrano et al., 1981; El Amrani et Chacon, 1996; Lamas et al., 2011), le Portugal (Alonso et al., 2010), le Maroc (Ejjaaouani et al., 2013; Mouhssine et al., 2015) et l'Algérie (Djedid et al., 2001; Bekkouche et al., 2002; Derriche et Cheikh-Lounis, 2004; Boudlal et al., 2014) pour ne citer que quelques pays du pourtour méditerranéen et pour les aspects comportementaux de ces terrains. En pratique, les géotechniciens se trouvent souvent confrontés à des terrains dont le comportement est intermédiaire entre celui des sols et des roches et qui sont de ce fait difficiles à traiter avec les approches classiques. Les trois comités français de géologie de l'ingénieur, de mécanique des sols et de mécanique des roches ont proposé six critères pour identifier les sols indurés et les roches tendres (SIRT) dans le cadre des études de sites naturels ou de projets de génie civil 
(Guilloux et al., 2005). Deux critères au moins doivent être vérifiés pour qu'un terrain soit déclaré comme SIRT. Les marnes sont considérées comme des sols indurés ou des roches tendres, car elles vérifient au moins quatre des six critères de définition des SIRT (résistance, porosité, minéralogie, évolutivité).

Au cours des années 1970 et 1980 est apparu la notion d'effets de structure dans les terrains naturels (Leroueil et Vaughan, 1990 ; Clayton et Serratrice, 1993). D'après Mitchell (1976), la structure d'un sol indique comment les particules individuelles s'agglomèrent, se lient entre elles et forment un ensemble qui incorpore de l'eau. En produisant ces effets de structure, les liaisons internes confèrent aux terrains un comportement analogue à celui des roches poreuses tendres. Généralement, cette structure engendre une amélioration des propriétés mécaniques du sol en termes de raideur et de résistance. Ces liaisons sont héritées de multiples facteurs. Les structures les plus simples se constituent pendant et après le dépôt dans les conditions d'un mécanisme de consolidation unidimensionnelle. D'autres types de structures postsédimentaires se forment en profondeur sous l'effet de processus divers de déchargement, de fluage, de thixotropie ou de cimentation et, plus généralement, de diagénèse. À l'inverse, des processus géologiques de nature différente sont à l'origine d'une réduction des effets de structure, par endommagement des liaisons et réarrangement de l'assemblage des grains sous l'action de déformations ou par l'effacement des liaisons sous l'effet de transformations physico-chimiques d'altération au sens large.

Les structures des sols raides et des roches tendres sont d'origines diverses. Mais les effets de structure ont en commun de conférer à ces terrains un comportement plutôt rigide et fragile à basse pression, analogue à celui observé dans les sols surconsolidés. Toutefois, il s'agit d'un phénomène différent. Les effets de structure déterminent donc le comportement des terrains naturels, au même titre que l'histoire du sol et sa porosité initiale. Par ailleurs, ces effets sont très progressifs, ce qui aboutit généralement à une très grande variété d'états et de nuances de comportement pour une même nature de sol au sein d'une même formation géologique.

En matière de comportement mécanique, les effets de structure se manifestent à l'échelle des éprouvettes de laboratoire et des échelles plus vastes au sein des massifs argileux, marneux ou rocheux. Ces effets prennent pour assise des phénomènes qui se produisent à l'échelle microscopique. La composition minéralogique et la microstructure conditionnent à des degrés divers les propriétés physiques et mécaniques de ces terrains. Cela s'explique par la nature variée des argiles, mais aussi par les processus physico-chimiques d'interaction de ces minéraux avec l'eau et la présence en proportions diverses de quartz, de carbonates (de calcite notamment) et d'autres constituants. Mais il n'est pas possible de dégager les rapports existant entre la minéralogie et les propriétés mécaniques des marnes sans chercher à mettre en évidence leur microstructure (Le Roux, 1971). Car l'observation de la microstructure (ou texture) vise à identifier l'arrangement spatial des particules et des agglomérats (ou agrégats), des grains et des vides, mais vise aussi à reconnaître la présence de dislocations et de microfissures, puis les orientations préférentielles de ces indices d'endommagement. Ainsi, de nombreux travaux expérimentaux ont été consacrés au recueil des propriétés microscopiques des roches argileuses en lien avec leurs propriétés mécaniques (Le Roux, 1972 ; Collins et Mc Gown, 1974; Delage et Lefebvre, 1984; Pejon et al., 1997; Fabre, 2005; Dehandschutter et al., 2005; Fabre et Pellet, 2006; Tran, 2014; Liu et al., 2016; parmi d'autres).

En se plaçant à une échelle macroscopique, beaucoup d'auteurs ont montré une divergence des comportements entre la réponse d'un sol testé dans son état naturel à la réponse du sol reconstitué, à commencer par Bishop (1971) pour l'argile de Londres. En compression oedométrique, la réponse du sol reconstitué se caractérise par une droite de compressibilité qui peut être considérée comme une référence (Burland, 1990). La comparaison de la réponse du sol chargé dans son état naturel à cette droite «intrinsèque» donne un moyen d'apprécier les effets de structure (Burland et al., 1996; Cotecchia et Chandler, 1997, 2000; Cotecchia et al., 2007; Amorosi et Rampello, 2007; Gasparre et al., 2007; Gasparre et Coop, 2008). Dans une démarche différente, qui présente l'avantage de ne pas avoir à reconstituer le sol, Amorosi et Rampello (2007) suggèrent de prendre comme référence le sol préalablement comprimé sous des fortes pressions isotropes qui sont de nature à détruire les effets de la structure initiale. Il faut recourir à des essais à haute pression pour cela, en capacité de dépasser l'état limite du sol.

Dans les sols raides, le cisaillement à basse pression produit la rupture du sol, avec des pics plus ou moins prononcés suivant l'état structuré du sol, accompagnés par l'apparition de fissures. La rupture est atteinte avant l'état critique. Sous des hautes pressions, les réponses s'apparentent à celles des argiles normalement consolidées où la rupture coïncide avec l'état critique, à condition d'exercer des pressions d'autant plus forte que le sol est structuré. Dans le même sens et sous les chargements à tendance isotrope, le seuil de préconsolidation, qui marque la transition vers le domaine plastique du sol, gagne en intensité avec le degré de structuration du sol. Finalement, un domaine pseudo-élastique s'établit dans l'espace des contraintes autour de l'état naturel du sol en place. Ce domaine est délimité par une surface d'état limite qui, à deux dimensions, adopte une forme plus ou moins elliptique (Crooks et Graham, 1976; Tavenas et Leroueil, 1977, 1979; Graham et al., 1983 ; Leroueil et Vaughan, 1990 ; Aversa et al., 1993). Toutefois, côté isotrope, cette frontière ne se présente pas comme une ligne étroite mais elle prend l'allure d'une bande plus ou moins large. Dans les sols argileux sensibles ou les roches poreuses tendres, l'ensemble des seuils qui constituent cette frontière sont caractérisés par de brusques variations de volume irréversibles. Mais, dans les sols argileux raides et compacts, les courbes de compressibilité présentent une courbure continue qui rend difficile l'identification d'une pression de préconsolisation en tant qu'état limite (Bishop et al., 1965; Burland 1990; Cotecchia et Chandler, 2000; Gasparre et al., 2007; Gasparre et Coop, 2008 ; Yang et al., 2014).

Beaucoup de sol indurés et de roches tendres présentent une structure anisotrope liée à leur dépôt, qui s'accompagne d'une anisotropie mécanique plus ou moins prononcée (Graham et Houlsby, 1983; Lings et al., 2000; Gasparre et al., 2007). Les marnes en général, et souvent les faciès les plus structurés parmi elles, n'échappent pas à cette forme de comportements. Dans les terrains faiblement anisotropes, l'anisotropie s'avère généralement plus exprimée sur les 
propriétés élastiques que sur les résistances. En d'autres termes, l'anisotropie s'estompe avec l'accumulation des déformations plastiques (Sivakumar et al., 2001).

Les argiles raides sont souvent fissurées avec des densités de fissuration variables et sous diverses formes, qui vont d'une fissuration latente jusqu'à une fissuration exprimée. Toutes ces discontinuités naturelles sont souvent héritées d'un déconfinement lié à l'histoire géologique (Skempton et al., 1969). Sinon, elles résultent des déformations de cisaillement qui ont été imprimées au massif, ou d'un mécanisme de retrait. À l'extrême, la fissuration peut aboutir à créer une structure totalement anisotrope comme celle des marnes schistosées par exemple. La présence de fissures produit un effet profond sur le comportement mécanique des terrains (Skempton et Petley, 1967; Skempton et al., 1969 ; Marsland, 1971 ; Costa Filho, 1984; Picarelli et al., 2006 ; Vitone et Cotecchia, 2011). Leur résistance est fortement influencée par les fissures préexistantes ou nouvellement formées. La fissuration rend les terrains sensibles aux effets de décompression et par conséquent aux effets du prélèvement (remaniement). À l'inverse, les chargements peuvent favoriser la déstructuration des terrains argileux. Callisto et Rampello (2004) montrent que les déformations volumiques plastiques contribuent à la dégradation progressive de la structure de trois argiles naturelles.

Les propriétés des terrains structurés sont changées par le processus d'altération pour aboutir à des sols pseudosurconsolidés dont les propriétés mécaniques sont dégradées par rapport aux propriétés initiales (Chandler, 1969, 1972; Chandler et Apted, 1988). Cafaro et Cotecchia (2001) montrent que l'altération s'interprète comme une dégradation des liaisons inter-particulaires et se traduit par une réduction de la raideur du sol et de sa résistance. Maquaire et al. (2003) montrent que l'altération des « Terres Noires » dans les Alpes est à l'origine de l'érosion et des ravinements des versants. Généralement, la progression de l'altération n'est pas uniforme et les massifs altérés possèdent des propriétés très dispersées et caractérisées par une grande variabilité spatiale.

Les différents points examinés ci-dessus concernent les comportements mécaniques des sols indurés et des roches tendres à dominante argileuse et plus spécialement les argiles raides et des marnes. Dans la suite, des illustrations de ces comportements sont examinées à une échelle macroscopique au travers d'exemples recueillis pendant de nombreux programmes d'essais en laboratoire réalisés dans le cadre de reconnaissances de massifs marneux pour des projets d'ouvrages en France. Cet aperçu s'adresse essentiellement aux propriétés de déformabilité et de résistance des terrains marneux homogènes tendres, homogènes indurés, fissurés ou altérés. La variété des comportements mécaniques ainsi observés montre la diversité des propriétés de ces terrains intermédiaires entre les argiles et les roches argileuses.

\section{Terrains oligocènes à Marseille}

\subsection{Résistance des terrains dans leur ensemble}

Le bassin sédimentaire tertiaire de Marseille est formé par des niveaux oligocènes comprenant principalement des marnes, mais aussi des grès et des poudingues, puis des dépôts quaternaires et des remblais en surface. Des tufs s'y rencontrent aussi. Les formations de l'Oligocène se caracté-

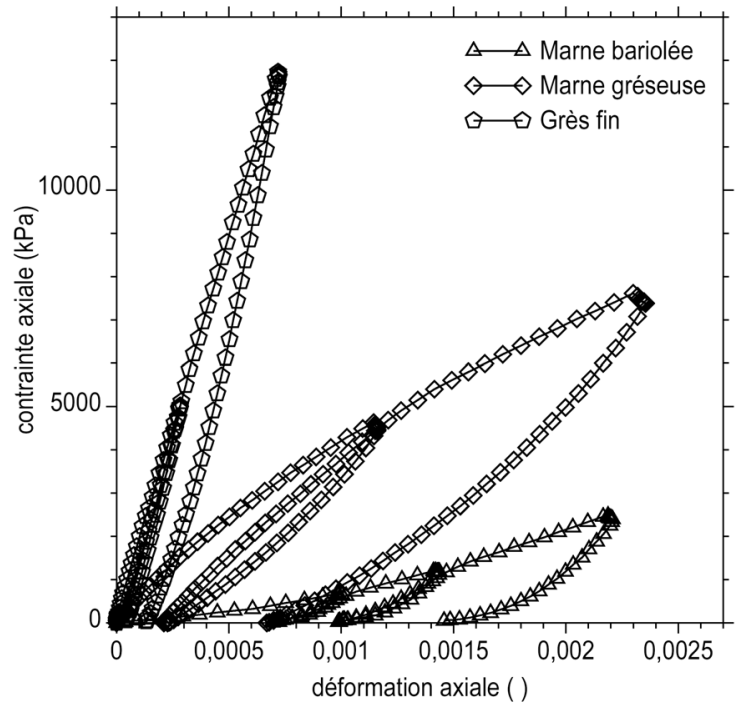

Fig. 1. Courbes contrainte déformation pour des essais de compression uniaxiale sur des marnes et des grès de l'Oligocène plus ou moins indurés.

Fig. 1. Stress-strain curves for unconfined compression tests on Oligocene marl and sandstone more or less indurated.

risent par la diversité de leurs faciès mais aussi la diversité de leurs propriétés physiques et mécaniques, en raison de l'hétérogénéité naturelle des matériaux en profondeur et leur altération en surface. Tous ces matériaux, et tout particulièrement les marnes, ont fait l'objet de programmes d'essais de laboratoire à l'occasion des reconnaissances géotechniques réalisées dans le cadre de projets d'ouvrages en surface ou souterrains. La description des terrains de l'Oligocène d'après l'observation visuelle des échantillons carottés a conduit à classer les terrains en familles de matériaux marneux à gréseux. Cette classification se trouve en bon accord avec les propriétés physiques et mécaniques mesurées. Les indices des vides moyens des terrains homogènes vont de 0,41 pour les marnes argileuses à 0,21 pour les grès marneux. Les marnes fissurées sont plus lâches avec un indice des vides moyen de 0,47. La dispersion est grande.

Les terrains de type rocheux (marnes gréseuses, grès, poudingues, tufs) sont caractérisés au moyen d'essais de compression uniaxiale et d'essais brésiliens. Les modules de Young sont mesurés à l'aide d'un extensomètre de précision disposé dans le tiers central des éprouvettes de diamètre $50 \mathrm{~mm}$ et d'élancement deux. Un exemple est donné sur la Figure 1 qui présente les courbes contrainte axiale et déformation axiale $\left(\varepsilon_{\mathrm{a}}\right.$, $\sigma_{\mathrm{a}}$ ) enregistrées sur trois éprouvettes pendant des cycles de compression uniaxiale. Les matériaux et leurs indices des vides sont respectivement : une marne bariolée $(e=0,45)$, une marne gréseuse $(e=0,34)$ et un grès fin $(e=0,17)$. La forme des courbes dépend nettement de la nature des terrains, comme leur compacité et leur déformabilité. Ici, les modules de Young initiaux tangents sont égaux à $0,5,5,2$ et $18 \mathrm{GPa}$ respectivement. L'hystérésis des courbes et les déformations irréversibles accumulées à chaque cycle dépendent aussi de la nature des matériaux.

Les graphiques de la Figure 2 montrent l'évolution des moyennes des résistances en compression uniaxiale $\sigma_{\mathrm{c}}$ des 

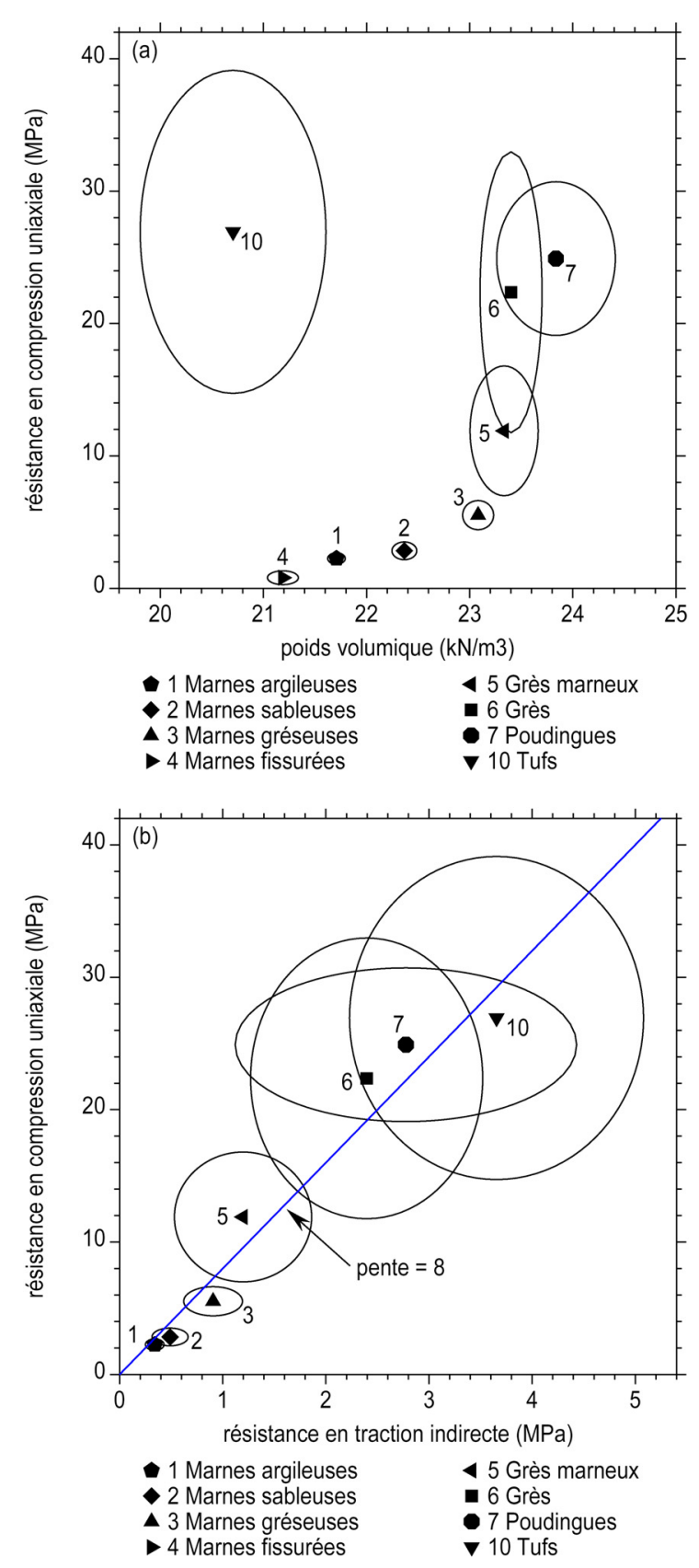

Fig. 2. Terrains de l'Oligocène. Relations entre les densités et les résistances. a) Résistances en compression uniaxiale en fonction des poids volumiques. b) Résistances en compression uniaxiale en fonction des résistances en traction indirecte. Intervalles de confiance à $95 \%$.

Fig. 2. Oligocène material. Relationship between densities and resistances. a) Unconfined compressive strength versus unit weight. b) Unconfined compressive strength versus indirect tensile strength. 95\% confidence interval.

différents terrains de l'Oligocène et des tufs quaternaires en fonction des poids volumiques $\gamma$ et des résistances en traction indirecte $\sigma_{\mathrm{tb}}$. Les terrains ont été classés visuellement dans les familles 4 (marnes fissurées), 1 (marnes argileuses), 2 (marnes sableuses), 3 (marnes gréseuses), 5 (grès marneux), 6 (grès), 7 (poudingues) et 10 (tufs), d'après la description qui est effectuée quand les échantillons carottés sont ouverts au laboratoire. Les échelles sont linéaires. Les intervalles de confiance à $95 \%$ sont reportés sous la forme d'ellipses autour des points moyens. À l'exclusion des tufs, la progression des résistances en compression uniaxiale prend une allure parabolique en fonction des poids volumiques (Fig. 2a). Les résistances en compression uniaxiale croissent quasi-linéairement en fonction des résistances en traction indirecte pour sept des huit familles de terrains (Fig. 2b). Les résistances en traction des marnes fissurées n'ont pas été mesurées. Les moyennes des résistances s'alignent approximativement sur une droite de pente huit. Ce rapport $\sigma_{\mathrm{c}} / \sigma_{\mathrm{tb}}=8$ est souvent observé.

La dispersion des mesures de résistance est forte dans chaque lot d'éprouvettes, comme toujours dans ces essais, avec des coefficients de variation rarement inférieurs à 0,7 et parfois supérieurs à 1,5. Cela tient beaucoup à l'allure des distributions des résistances qui sont systématiquement de forme exponentielle ou gamma. Des exemples seront présentés dans la suite (Fig. 9). Néanmoins, il est intéressant de constater que le classement des roches de l'Oligocène d'après leurs résistances $\sigma_{\mathrm{c}}$ et $\sigma_{\mathrm{tb}}$, pour l'échantillonnage disponible ici, s'établit en familles distinctes et conformes à la description visuelle de ces terrains et conformes à leurs caractéristiques d'état. L'analyse des données montre aussi que cette classification est vérifiée au fil du temps pour les campagnes successives de reconnaissances qui s'adressent à des sites différents et éloignés les uns des autres dans le bassin de Marseille (Serratrice, 1998).

\subsection{Résistance des marnes les plus tendres}

Les programmes expérimentaux consacrés aux terrains de l'Oligocène concernent surtout les marnes les plus tendres. $\mathrm{Au}$ laboratoire, ces matériaux se découpent manuellement au touret ou à la trousse, par opposition à des terrains plus indurés qui ne peuvent être découpés que par carottage (grès, grès marneux) et qui sont caractérisés mécaniquement au moyen d'essais de type roche (Fig. 2). Il s'agit donc des marnes des familles 1 (marnes argileuses), 2 (marnes sableuses) et 4 (marnes fissurées). Les propriétés mécaniques de ces terrains ont été obtenues à l'aide d'essais triaxiaux à basse pression, dans la gamme $0-1 \mathrm{MPa}$, ou à haute pression dans la gamme 1$2 \mathrm{MPa}$. Les essais triaxiaux sont réalisés à l'aide de différentes unités triaxiales conventionnelles ou asservies, à basse pression et à haute pression (Serratrice, 2002).

Les éprouvettes triaxiales sont découpées manuellement au touret au cœur des échantillons carottés (diamètre initial $d_{0}=50 \mathrm{~mm}$, élancement deux). Les essais triaxiaux comprennent trois phases principales : saturation par paliers pour atteindre une contre-pression comprise entre 300 à $500 \mathrm{kPa}$, consolidation isotrope sous la contrainte moyenne effective $p_{c}$ comprise entre 0,3 et $12 \mathrm{MPa}$, cisaillement en compression ou en extension, en condition drainée ou non drainée, à des vitesses de cisaillement comprises entre 1 et $4 \mu \mathrm{m} / \mathrm{min}$ (de $110^{-7}$ à $710^{-7} \mathrm{~s}^{-1}$ pour une éprouvette de $100 \mathrm{~mm}$ de hauteur). Les mesures enregistrées sont exprimées au moyen de la déformation axiale $\varepsilon_{a}$, la déformation volumique $\varepsilon_{v}$, la pression interstitielle $u$, la pression moyenne effective $p=\left(\sigma_{\mathrm{a}}^{\prime}+2 \sigma_{\mathrm{r}}{ }_{\mathrm{r}}\right) / 3$ et le déviateur $q=\sigma_{\mathrm{a}}{ }^{\prime}-\sigma_{\mathrm{i}}{ }_{\mathrm{i}}$, où $\sigma_{\mathrm{a}}{ }_{\mathrm{a}}$ et la contrainte axiale effective et $\sigma_{\mathrm{r}}$ la contrainte radiale effective. 


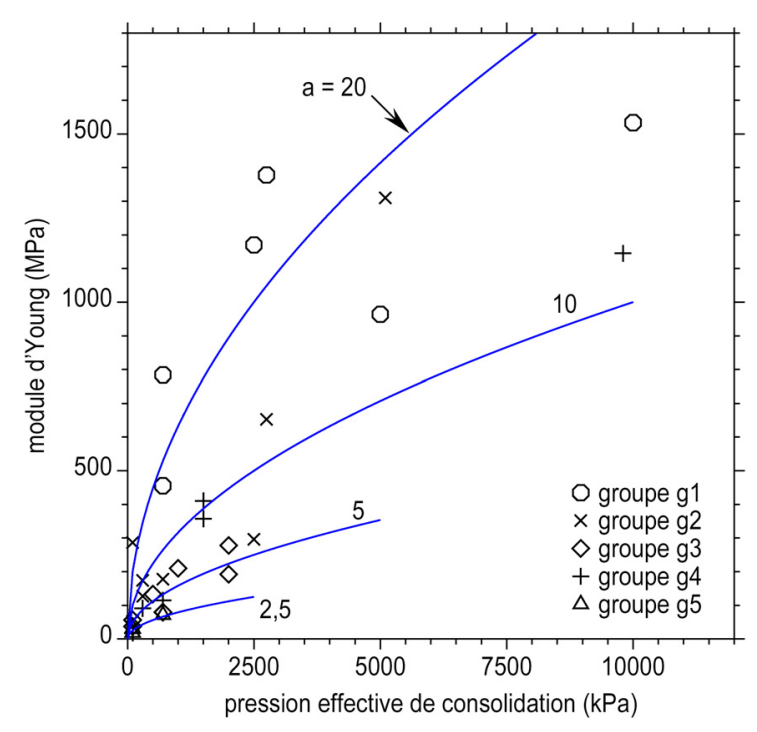

Fig. 3. Essais triaxiaux drainés à haute pression (CD HP). Modules initiaux tangents en compression pour les cinq groupes g1 à g5 des marnes les plus tendres.

Fig. 3. High pressure drained triaxial tests (CD HP). Initial tangent modulus in compression for the five subgroups of softer marls.

Les pentes initiales des courbes contrainte-déformation $\left(\varepsilon_{\mathrm{a}}, q\right)$ représentent des modules de Young initiaux tangents $(E=\Delta q$ $\left(\Delta \varepsilon_{\mathrm{a}}\right)$. Ils sont déterminés sur la partie rectiligne initiale des courbes $\left(\varepsilon_{\mathrm{a}}, q\right)$ pour une déformation axiale égale à 0,005 environ $(0,5 \%)$. Les résistances sont définies sur les chemins des contraintes drainés ou non drainés, en appliquant le critère de rapport de résistance maximal $(q / p)_{\max }$.

Malgré cette limitation des programmes expérimentaux aux marnes les plus tendres, les essais triaxiaux aboutissent à une large variété de résistances et une grande dispersion. Il s'est avéré nécessaire d'établir une sous-classification de ces terrains d'après leurs résistances. Cinq groupes (ou sousfamilles) ont été définis pour représenter la progression des résistances des marnes homogènes en allant des marnes les plus résistantes et les plus raides vers les marnes les moins résistantes et les plus tendres (de g1 à g4), puis les marnes fissurées (g5).

La Figure 3 montre l'évolution des modules de Young initiaux tangents en compression en fonction de la pression effective de consolidation isotrope $p_{c}$ pendant les essais triaxiaux drainés à haute pression. Les modules augmentent avec les pressions de confinement. Une interprétation est proposée avec un accroissement de forme parabolique des modules de Young avec la pression $p_{c}$ suivant l'expression $\left(p_{c}\right.$ en $\mathrm{kPa}$ et $E$ en $\left.\mathrm{MPa}\right)$ :

$$
E=a_{1}\left(p_{c} / p_{u}\right)^{0,5}+b_{1},
$$

où $a_{1}$ vaut $20,10,5$ et $2,5 \mathrm{MPa}$ pour les groupes g1, g2, g3 et g4 respectivement, $a_{1}=2,5 \mathrm{MPa}$ pour le groupe $\mathrm{g} 5, b_{1}=0$ dans tous les cas et $p_{\mathrm{u}}$ la pression unité $\left(p_{\mathrm{u}}=1 \mathrm{kPa}\right)$. Des expressions de ce type sont admises couramment (Houlsby et Wroth 1991; Biarez et Hicher 1994).
Les points à la rupture par cisaillement $\left(p_{\mathrm{r}}, q_{\mathrm{r}}\right)$ en condition drainée et non drainée mesurés pendant les essais triaxiaux réalisés sur les marnes et d'après le critère $(q / p)_{\max }$ sont représentés sur la Figure 4 dans les plans de contraintes effectives $(p, q)$ à différentes échelles. Il apparaît une grande dispersion des résistances sur toute l'étendue des graphiques, bien que les marnes testées appartiennent aux trois familles des terrains les moins résistants (argileuses, sableuses et fissurées des familles 1, 2 et 4, groupes g1 à g5). La sous-classification g1 à $\mathrm{g} 5$ repose sur le constat que le rapport des contraintes à la rupture $\eta_{\mathrm{r}}=q_{\mathrm{r}} / p_{\mathrm{r}}$ augmente quand l'indice des vides initial $e_{0}$ diminue. Les marnes les plus compactes sont en moyenne les plus résistantes.

La représentation des points à la rupture $\left(p_{\mathrm{r}}, q_{\mathrm{r}}\right)$ dans les plans des contraintes effectives $(p, q)$ de la Figure 4 montre aussi que les enveloppes de rupture sont courbes à basse pression, sauf le groupe g5 des marnes les moins résistantes dont les résistances s'alignent à peu près sur une droite passant par l'origine du plan $(p, q)$. Cette droite a pour pente $M_{\mathrm{c}}=0,87$. Il lui correspond un angle de frottement interne $\varphi^{\prime}=22,1$ degrés. Cet angle de frottement est confirmé par les essais triaxiaux en extension, dont les points à la rupture à haute pression s'alignent sur une enveloppe droite de pente $M_{\mathrm{e}}=-0,67$ (essais non présentés ici).

La courbure des enveloppes de rupture à basse pression est assez souvent observée (Croce et al., 1969; Tavenas et Leroueil, 1977, 1979; Graham et al., 1983 ; Serratrice, 1995 ; Burland et al., 1996). Ici, les enveloppes de rupture des familles g1 à g4 ont été représentées par des arcs de spirales logarithmiques d'équations :

$$
q=\left(-a_{2} \ln \left(p / p_{u}\right)+b_{2}\right) p
$$

où $a_{2}$ et $b_{2}$ sont des paramètres et $p_{\mathrm{u}}$ la pression unité $\left(p_{\mathrm{u}}=1 \mathrm{kPa}\right)$. Ces arcs de courbes sont limités au domaine: $p_{\mathrm{u}}<p$ et $M_{\mathrm{c}}<q<3 p$. Les paramètres $a_{2}$ et $b_{2}$ s'identifient facilement par régression à partir de la représentation des données dans le plan $\left(\lg p_{\mathrm{r}}, \eta_{\mathrm{r}}\right)$ où $\eta_{\mathrm{r}}=q_{\mathrm{r}} / p_{\mathrm{r}}$ est le rapport des contraintes de cisaillement à la rupture. Puis, il a été admis aussi que, à très haute pression, les enveloppes de rupture de chacune des familles de marne retrouvent une allure linéaire dont les pentes $M_{\mathrm{c}}$ augmentent dans chacun de groupes g4 à g1 tout en conservant des cohésions nulles. Ces droites sont représentées par des segments dans les plans des contraintes effectives $(p, q)$ de la Figure 4. Les spirales logarithmiques se raccordent à ces segments. Cette interprétation se heurte à la limite de capacité de l'appareillage. Des pressions plus fortes devraient être appliquées pour confirmer ces enveloppes de rupture, qui gagnent rapidement en étendue avec l'effet de l'induration.

Le Tableau 1 récapitule les caractéristiques des enveloppes de rupture pour chacun des cinq sous-groupes de marnes. Les pentes $M_{\mathrm{c}}$ et $M_{\mathrm{e}}$ sont différentes en valeurs absolues pour un même angle de frottement $\varphi^{\prime}$, car elles représentent les branches opposées du critère de Coulomb appliqué à ces essais triaxiaux (en symétrie de révolution) à haute pression. À basse pression, des critères de Coulomb tangents ou sécants à ces critères courbes sont à définir dans des plages de pressions moyennes données pour évaluer la cohésion d'un des sousgroupes de marne. 

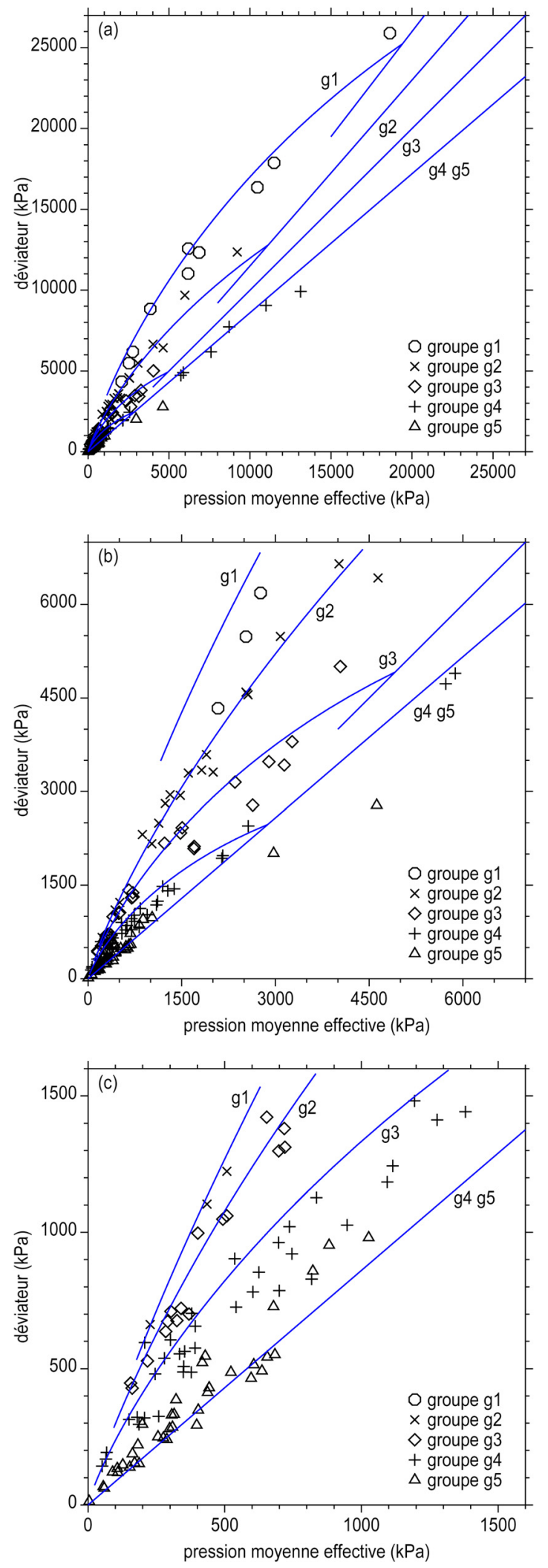

Fig. 4. Enveloppes de rupture emboîtées des marnes de l'Oligocène plus ou moins indurées. a) b) c) Plans des contraintes effectives (p, q) à différentes échelles.

Fig. 4. Failure envelopes fitted together for Oligocene marl more or less indurated. a) b) c) Effective stress plan $(p, q)$ at various scales.
Tableau 1. Propriétés des enveloppes de rupture des marnes tendres. Table 1. Strength envelops parameters of soft marls.

\begin{tabular}{lllllll}
\hline Sous-groupe & $\begin{array}{l}e_{0} \text { moyen } \\
(-)\end{array}$ & $\begin{array}{l}M_{c} \\
(-)\end{array}$ & $\begin{array}{l}M_{e} \\
(-)\end{array}$ & $\begin{array}{l}\varphi^{\prime} \\
\left({ }^{\circ}\right)\end{array}$ & $\begin{array}{l}a_{2} \\
\left(^{\circ}\right)\end{array}$ & $\begin{array}{l}b_{2} \\
\left(^{\circ}\right.\end{array}$ \\
\hline g1 & 0,341 & 1,30 & $-0,91$ & 32,3 & 0,60 & 7,26 \\
g2 & 0,333 & 1,15 & $-0,83$ & 28,9 & 0,45 & 5,32 \\
g3 & 0,371 & 1,00 & $-0,75$ & 25,4 & 0,51 & 5,30 \\
g4 & 0,448 & 0,86 & $-0,67$ & 22,1 & 0,45 & 4,43 \\
g5 & 0,497 & 0,86 & $-0,67$ & 22,1 & - & - \\
\hline
\end{tabular}

\section{Autres exemples de marnes homogènes tendres}

\subsection{Compression}

Le second exemple concerne une marne du Pliocène prélevée par blocs pendant le creusement du tunnel sud de Las Planas dans la région de Nice (Lee et al., 1993). La marne est homogène, consistance, non fissurée, de couleur grise. Son indice des vides moyen vaut 0,40 . Les résultats des phases de cisaillement triaxial drainé en compression à basse pression et haute pression sont présentés sur la Figure 5 avec les courbes contrainte-déformation $\left(\varepsilon_{\mathrm{a}}, q\right)$, les chemins des contraintes effectives $(p, q)$ et les chemins des déformations $\left(\varepsilon_{\mathrm{a}}, \varepsilon_{\mathrm{v}}\right)$, où $\varepsilon_{\mathrm{v}}$ est la déformation volumique. Les modules de Young $E$ augmentent avec la pression isotrope effective de consolidation $p_{\mathrm{c}}$, comme le montre le graphique de la Figure 6. L'accroissement de $E$ avec $p_{\mathrm{c}}$ s'interprète avec la relation empirique (1), $a_{1}=6,82 \mathrm{MPa}$ et $b_{1}=0$ ( $p_{\mathrm{c}}$ en $\mathrm{kPa}$ et $E$ en $\mathrm{MPa}$ ). La pente initiale $\mu=\Delta \varepsilon_{\mathrm{v}} / \Delta \varepsilon_{\mathrm{a}}$ des chemins de déformation $\left(\varepsilon_{\mathrm{a}}, \varepsilon_{\mathrm{v}}\right)$ permet de mesurer le coefficient de Poisson qui vaut $v=0,15$ environ, indépendamment de la pression de consolidation, d'après la relation suivante (Fig. $5 \mathrm{c}, \mu \approx 0,70$ environ) :

$$
v=(1-\mu) / 2 \text {. }
$$

Les courbes $\left(\varepsilon_{\mathrm{a}}, \mathrm{q}\right)$ présentent des pics de résistance à basse pression, qui s'estompent progressivement avec l'accroissement des pressions de confinement (Fig. 5a). Des pics de contractance-dilatance apparaissent le long des chemins de déformation $\left(\varepsilon_{\mathrm{a}}, \varepsilon_{\mathrm{v}}\right)$ vers $1 \%$ de déformation axiale et avant les pics de résistance à basse pression (Fig. $5 \mathrm{c}$ ). Après ces pics, la dilatance $\delta$ représente la pente de ces chemins $\left(\delta=\Delta \varepsilon_{\mathrm{v}} / \Delta \varepsilon_{\mathrm{a}}\right.$, $\delta<0$ pour une dilatance). La marne est fortement dilatante à faible pression moyenne. Au contraire, elle est continûment contractante à partir de $2 \mathrm{MPa}$ de confinement.

Dans le plan des contraintes effectives $(p, q)$ et sous les hautes pressions, l'enveloppe de rupture peut être assimilée à une droite passant par l'origine du plan et de pente $M_{\mathrm{c}}=1,18$ qui correspond aux caractéristiques $c^{\prime}=0$ pour la cohésion et $\varphi^{\prime}=29,5^{\circ}$ pour l'angle de frottement interne (Fig. 5b). À basse pression, la marne possède un supplément de résistance par rapport à cette droite, accompagné par des pics et de la dilatance, et qui se manifeste par une enveloppe de résistance courbe. En moyenne et comptetenu de la dispersion des résistances, une approximation linéaire tangente de cette enveloppe est proposée ici, qui possède les propriétés $c^{\prime}=370 \mathrm{kPa}$ et $\varphi^{\prime}=35,4^{\circ}$. D'autres approximations pourraient être adoptées sur des plages de pressions différentes, 

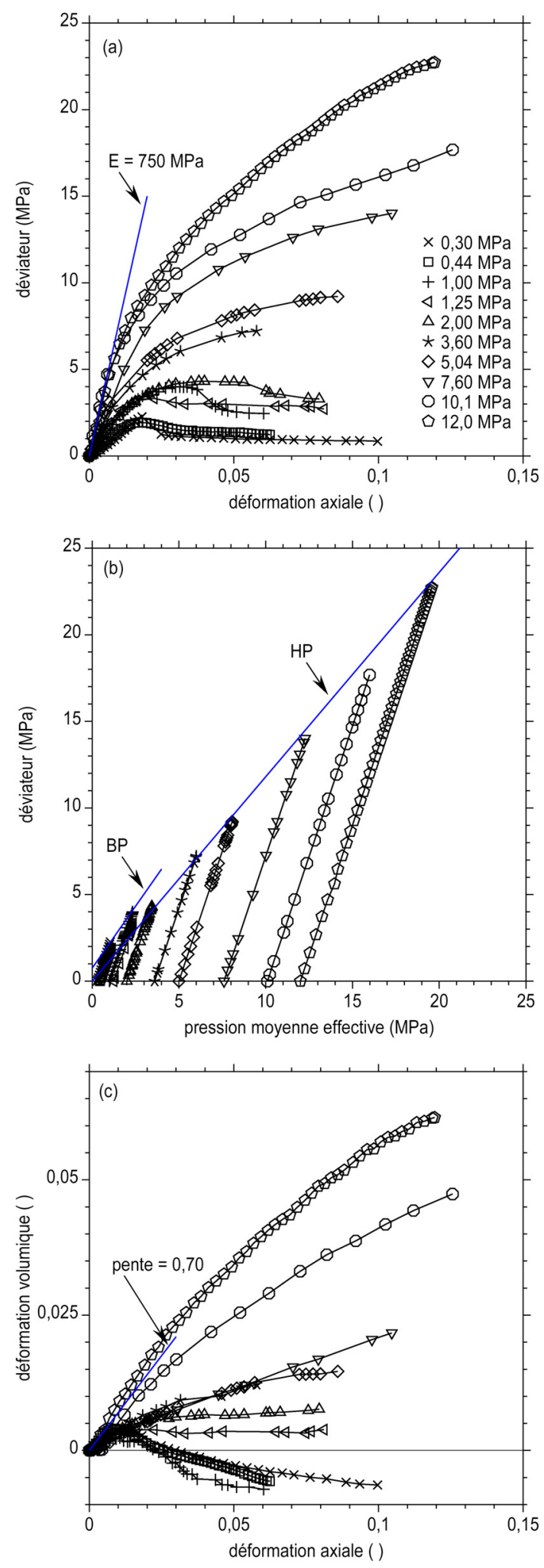

Fig. 5. Essais triaxiaux drainés à haute pression CD HP sur une marne homogène compacte. Phases de cisaillement en compression. a) Courbes contrainte-déformation. b) Chemins des contraintes effectives. c) Chemins des déformations.

Fig. 5. High pressure drained triaxial tests CD HP on homogeneous compact marl. Shear phase in compression. a) Stress-strain curves. b) Effective stress paths. c) Strain paths.

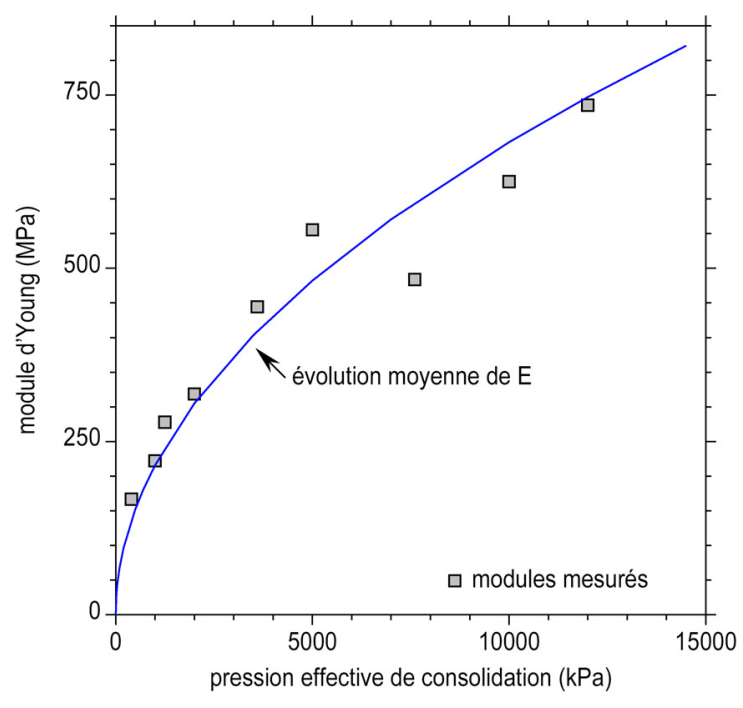

Fig. 6. Modules de Young initiaux tangents en fonction de la pression effective de consolidation au début des phases de cisaillement drainé à haute pression.

Fig. 6. Initial tangent modulus versus effective consolidation pressure at the beginning of drained compression loading at high pressure.

donnant des couples $c^{\prime}$ et $\varphi$ ' légèrement différents. Cette interprétation est semblable à celle de la Figure 4. En réalité, cette forme d'enveloppe de rupture, courbe à basse pression et rectiligne à haute pression, ne s'observe pas systématiquement. Cette partie rectiligne peut être courbe à son tour à très haute pression. Dans d'autres cas, une courbure continue s'établit, comme dans les roches. Cela concerne les marnes indurées à très indurées. Un exemple sera présenté dans la suite (Fig. 7a).

\subsection{Anisotropie}

Des essais de laboratoire ont été réalisés sur une marne compacte, grise et stratifiée, prélevée par carottage à l'occasion du creusement d'un tunnel dans la région d'Avignon (site de Bompas). Le matériau prélevé est une marne miocène, silteuse à sableuse, micacée, gris bleu, parfois fortement litée. La stratification est horizontale au sein du massif. Les sondages ont été réalisés en différents points du tracé du tunnel, verticalement dans le radier (perpendiculairement au litage) et horizontalement au front (dans le plan du litage). L'indice des vides moyen de la marne est égal à 0,35 . Des essais de compression uniaxiale ont été réalisés de manière systématique sur des éprouvettes d'élancement deux, simplement débitées $(86 \mathrm{~mm}$ de diamètre) ou carottées $(50 \mathrm{~mm}$ de diamètre) dans les échantillons. Plusieurs tentatives de découpage des éprouvettes ont échoué en raison de la présence de lits sableux, qui constituent des plans de faiblesse. La résistance en compression uniaxiale $\sigma_{c}$ est plus forte en moyenne dans les échantillons verticaux avec $\sigma_{c v}=3,56 \mathrm{MPa}$ (perpendiculaires au litage) que dans les échantillons horizontaux avec $\sigma_{\mathrm{ch}}=2,95 \mathrm{MPa}$ (parallèles au litage), avec des coefficients de variation supérieurs à 0,2 pour une moyenne générale $\sigma_{\mathrm{c}}=3,37 \mathrm{MPa}$ ( $h$ direction horizontale, $v$ direction verticale). Cette anisotropie mécanique de la marne est héritée de son anisotropie de structure. Le rapport des 

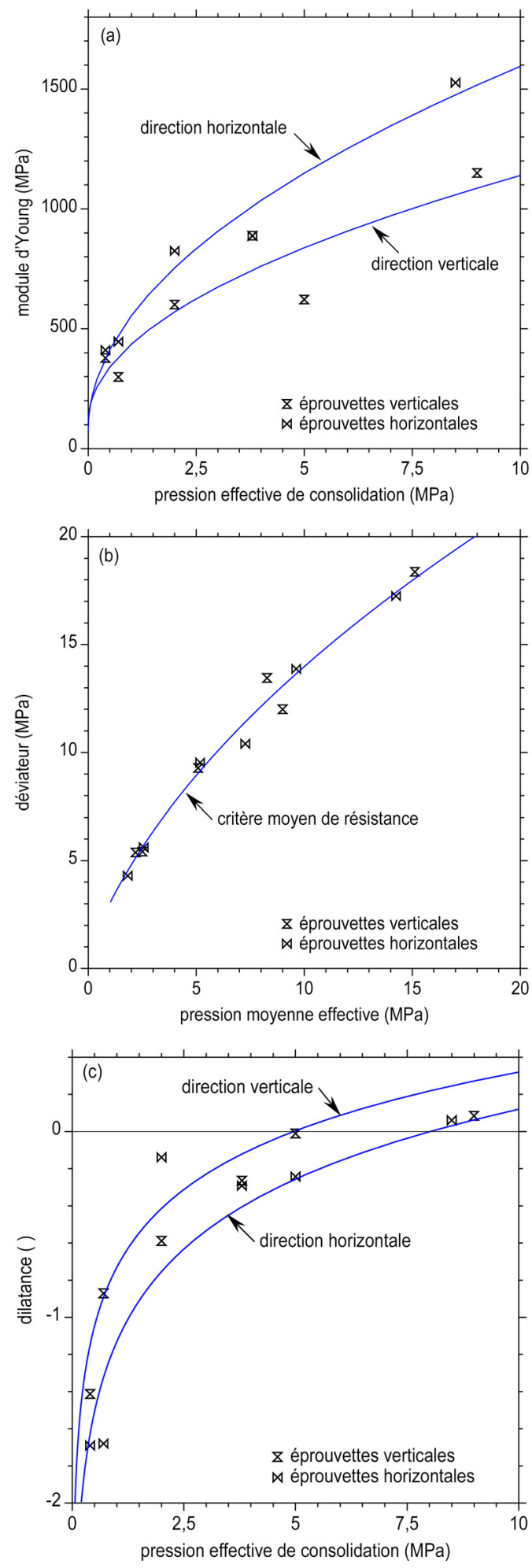

Fig. 7. Essais triaxiaux drainés à haute pression sur une marne litée. Éprouvettes horizontales et verticales. a) Modules de Young initiaux tangents en fonction de la pression effective de consolidation. b) Enveloppe de rupture. c) Dilatances.

Fig. 7. High pressure drained triaxial tests on a layered marl. Horizontal and vertical specimens. a) Initial tangent Young modulus versus effective consolidation pressure. b) Failure envelopes. c) Dilatancy slopes. résistances moyennes dans les deux directions du massif $\sigma_{\mathrm{ch}} /$ $\sigma_{\mathrm{cv}}=0,83$. Il diffère de l'unité.

Des essais triaxiaux drainés à haute pression ont été réalisés sur des éprouvettes de marne découpées dans les deux directions de prélèvement. Les modules initiaux tangents mesurés au début des chargements en compression augmentent avec la pression isotrope effective $p_{\mathrm{c}}$. Ils sont représentés sur la Figure 7a. Les éprouvettes verticales (dont l'axe est perpendiculaire au litage) possèdent des modules $E_{v}$ plus faibles que les éprouvettes horizontales de module $E_{\mathrm{h}}$ (dont l'axe est parallèle au litage). L'interprétation proposée donnant un accroissement de $E$ avec la racine carrée de $p_{c}$ est renouvelée ici (1). Les deux courbes représentées sur le graphique ont pour paramètres $a_{1}=10,3 \mathrm{MPa}, b_{1}=110 \mathrm{MPa}$ pour la direction verticale et $a_{1}=15,2 \mathrm{MPa}, b_{1}=74 \mathrm{MPa}$ pour la direction horizontale ( $p_{\mathrm{c}}$ en $\mathrm{kPa}$ et $E$ en $\mathrm{MPa}$ ). Le rapport $n=E_{\mathrm{h}} / E_{\mathrm{v}}$ vaut 1,5 environ. Il est à peu près inverse au rapport des résistances $\sigma_{\mathrm{ch}} / \sigma_{\mathrm{cv}}$

Les coefficients de Poisson déduits des pentes $\mu$ des chemins de déformations $\left(\varepsilon_{a}, \varepsilon_{v}\right)$ au début des chargements valent $v_{\mathrm{v}}=0,22$ pour les éprouvettes verticales et $v_{h}=0,18$ pour les éprouvettes horizontales, d'après l'équation (3). Les dilatances $\delta$ mesurées en fin de cisaillement diminuent en valeur absolue avec l'accroissement de la pression moyenne effective et sont plus grandes en valeur absolue pour les éprouvettes horizontales (axe parallèle au litage). Les éprouvettes horizontales sont plus dilatantes que les éprouvettes verticales (axe perpendiculaire au litage), comme le montre la Figure 7c. Mais la dispersion est grande. La marne ne devient contractante pendant les cisaillements qu'avec des pressions de consolidation comprises entre 5 et 7,5 $\mathrm{MPa}$.

Les points à la rupture $\left(p_{\mathrm{r}}, q_{\mathrm{r}}\right)$ en contraintes effectives sont reportés sur le graphique de la Figure $7 \mathrm{~b}$. Les résistances des éprouvettes verticales et horizontales sont proches. Il apparaît que, à la dispersion des mesures près, l'enveloppe de rupture est courbe avec une anisotropie finalement peu marquée contrairement à la tendance donnée par les compressions uniaxiales. L'anisotropie semble s'estomper vers les hautes pressions. Il faut noter que l'échantillonnage des éprouvettes n'est pas comparable entre les essais de compression uniaxiale (29 éprouvettes) et les essais triaxiaux à haute pression (deux éprouvettes par niveau de pression). Les éprouvettes sont découpées dans des échantillons différents, eux-mêmes répartis le long du tracé du tunnel. Ainsi, la dispersion des résistances mesurées ne permet pas de distinguer les deux directions de prélèvement en termes de résistances à haute pression. Bishop et al. (1965) constataient une réponse comparable pour l'argile de Londres.

Il est proposé d'interpréter les résistances de la marne litée au moyen d'un critère de rupture parabolique de Hoek et Brown (1980), qui s'écrit:

$$
q=\sigma_{a}^{\prime}-\sigma_{r}^{\prime}=\sigma_{c i}^{\prime}\left(m_{b} \sigma_{r}^{\prime} / \sigma_{c i}^{\prime}+s\right)^{0,5},
$$

où $\sigma^{\prime}{ }_{\mathrm{a}}$ et $\sigma^{\prime}{ }_{\mathrm{r}}$ sont les contraintes effectives axiales et radiales, $\sigma_{c i}$ est la résistance en compression uniaxiale, $m_{\mathrm{b}}$ et $\mathrm{s}$ deux paramètres. Pour la marne non fissurée dans son état naturel, ce qui est le cas ici, $s=1$. Il reste à déterminer $\sigma^{\prime}{ }_{\mathrm{ci}}$ et $m_{\mathrm{b}}$. Le critère s'écrit aussi $q^{2}=m_{\mathrm{b}} \sigma^{\prime}{ }_{\mathrm{ci}} \sigma_{\mathrm{r}}+\sigma^{\prime}{ }_{\mathrm{ci}}{ }^{2}$, ce qui permet d'identifier les deux paramètres $\sigma_{\text {ci }}^{\prime}$ et $m_{\mathrm{b}}$ à partir des coefficients $a$ et $b$ de 
la droite $q^{2}=a_{3} \sigma_{\mathrm{r}}+b_{3}$ qui exprime le critère dans le plan $\left(\sigma_{\mathrm{r}}{ }_{\mathrm{r}}\right.$, $q^{2}$ ), avec $a_{3}=m_{\mathrm{b}} \sigma^{\prime}{ }_{\mathrm{ci}}$ et $b_{3}=\sigma_{\mathrm{ci}}{ }^{2}$. Le report des données expérimentales dans ce plan aboutit avec la droite $q^{2}=34,7$ $\sigma^{\prime}{ }_{\mathrm{r}}+9,5\left(q\right.$ et $\sigma_{\mathrm{r}}$ en $\left.\mathrm{MPa}\right)$, soit $\sigma_{\mathrm{ci}}{ }_{\mathrm{c}}=3,16 \mathrm{MPa}$ et $m_{\mathrm{b}}=11,1 \mathrm{La}$ résistance $\sigma_{c i}$ est proche de la valeur moyenne mesurée en compression uniaxiale. Le critère de Hoek et Brown calculé avec ces paramètres et représenté dans le plan $(p, q)$ de la Figure $7 \mathrm{~b}$. La dispersion des résistances ne permet pas de distinguer les éprouvettes verticales des éprouvettes horizontales et le critère adopté ici, unique et moyen, révèle une faible anisotropie de la marne litée à la rupture et sous forte pression. Des critères de Coulomb tangents ou sécants à ce critère peuvent être déterminés alors, dans des plages de pressions moyennes effectives à définir.

Enfin, le report des dilatances $\delta=\Delta \varepsilon_{\mathrm{v}} / \Delta \varepsilon_{\mathrm{a}}$ en fonction des pressions effectives de consolidation $p_{\mathrm{c}}$ sur la Figure $7 \mathrm{c}$ montre des dilatances plus fortes en valeurs absolues dans la direction horizontale (axe parallèle au litage) que dans la direction verticale (axe perpendiculaire au litage). Une interprétation de ces variations est indiquée, de la forme $\delta=a_{4} \lg \left(p_{\mathrm{c}} / p_{\mathrm{u}}^{\prime}\right)+b_{4}$ avec $a_{4}=1,25$ est $b_{4}=-1,13$, puis $a_{4}=1,05$ et $b_{4}=-0,73$ pour les directions horizontales et verticales respectivement $\left(p_{\mathrm{c}}\right.$ en $\mathrm{MPa}$ et $p_{\mathrm{u}}=1 \mathrm{MPa}$ ).

\section{Marnes homogènes indurées}

\subsection{Déformabilité et résistance}

Les reconnaissances géotechniques réalisées dans le cadre des projets autoroutiers dans les Alpes ont amené à étudier plus en détail les propriétés des marnes dites «Terres Noires » $\mathrm{du}$ Jurassique moyen à supérieur. Ces terrains occupent de vastes espaces dans les vallées du Buëch et de la Durance dans le département des Hautes Alpes. Les «Terres Noires » forment un ensemble sédimentaire épais composé de marnes sombres et de petits bancs carbonatés et de marnes schisteuses de teinte sombre. Les marnes ont été prélevées par carottage dans de nombreux sondages très éloignés les uns des autres. Les marnes sont indurées et très compactes avec un indice des vides moyen égal à 0,12 . Leur teneur en carbonate de calcium varie de 15 à $50 \%$.

La caractérisation mécanique de ces terrains s'effectue principalement au moyen d'essais de compression uniaxiale et d'essais de traction indirecte (essais brésiliens). L'exemple d'une courbe contrainte déformation $\left(\varepsilon_{\mathrm{a}}, \sigma_{\mathrm{a}}\right)$ est donné sur la Figure $8 \mathrm{a}$. La déformation axiale $\varepsilon_{\mathrm{a}}$ est mesurée à l'aide d'un extensomètre de précision au cours de plusieurs cycles de chargement-déchargement (mesure locale de la déformation, vitesse de déformation axiale $10^{-5} \mathrm{~s}^{-1}$ ). Une hystérésis notable apparaît à chaque cycle, accompagnée par une réduction progressive du module de Young sécant, avec une accumulation graduelle de déformations irréversibles. Il n'y a pas de serrage. Le graphique de la Figure $8 \mathrm{~b}$ montre le module de Young tangent tiré de la courbe précédente par dérivation directe. Les trois phases de déchargement sont représentées par les segments $a-a 1, b-b 1$ et $c-c 1$. Chaque inversion du chargement a pour effet de restaurer le module tangent à sa valeur initiale ( $3 \mathrm{GPa}$ environ). Mais la courbe maîtresse m$\mathrm{m} 1$, qui serait obtenue par un chargement monotone et le long de laquelle se développent les phases de chargement pendant les cycles, montre la dégradation progressive du module
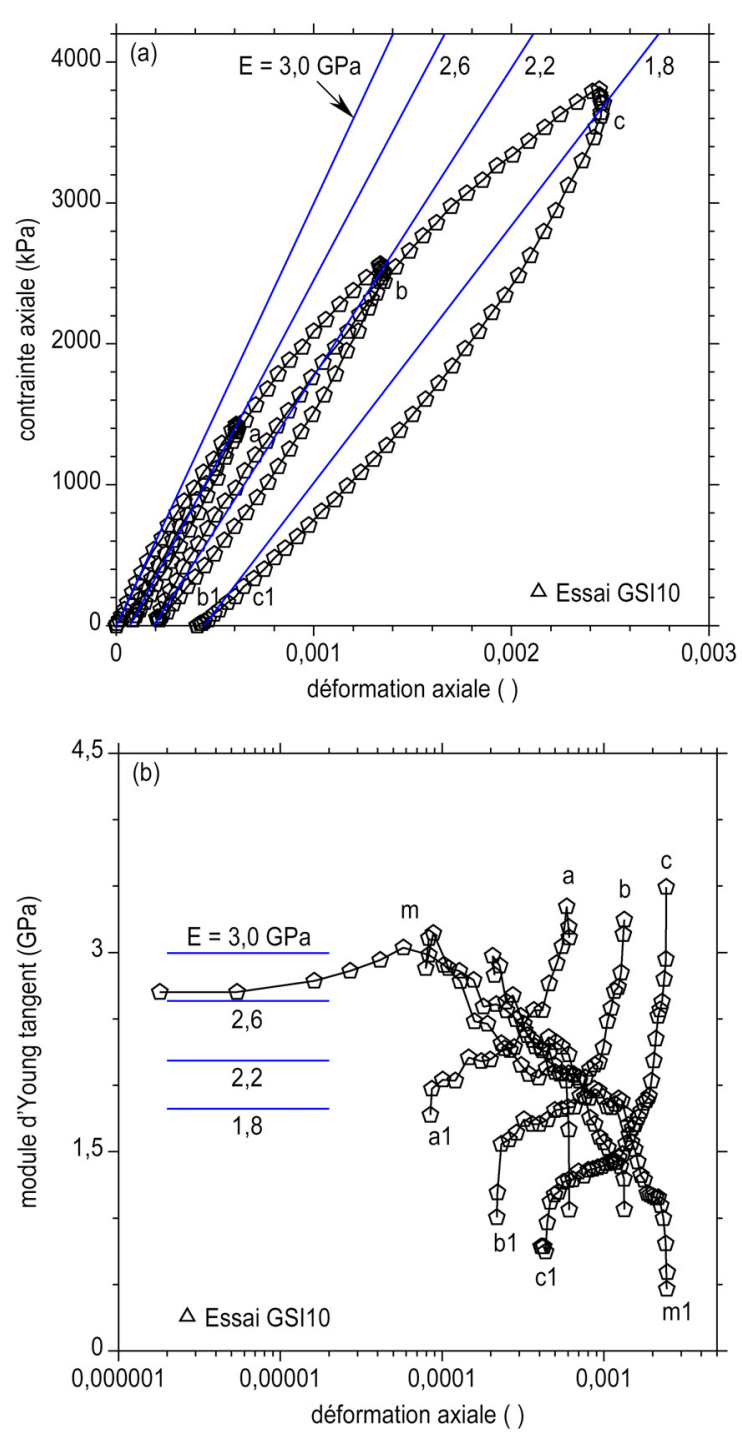

Fig. 8. Essais de compression uniaxiale réalisés sur des marnes indurées des «Terres Noires ». a) Courbes contrainte-déformation $\left(\varepsilon_{\mathrm{a}}\right.$, $\left.\sigma_{\mathrm{a}}\right)$. b) Module de Young tangent en fonction de la déformation axiale. Fig. 8. Unconfined compressive tests on indurated marls "Terres Noires". a) Stress-strain curves $\left(\varepsilon_{a}, \sigma_{a}\right)$. b) Tangent Young modulus versus axial strain.

tangent de la marne avec l'accumulation des déformations irréversibles. Le module initial tangent et les modules sécants associés aux trois cycles sont les pentes des droites représentées sur la Figure $8 \mathrm{a}(3,0,2,8,2,2$ et 1,8 GPa). Ils sont indiqués comme ordonnées sur la Figure $8 \mathrm{~b}$. En résumé, les courbes contrainte-déformation $\left(\varepsilon_{\mathrm{a}}, \sigma_{\mathrm{a}}\right)$ de la Figure 8 font apparaître un comportement typique des marnes indurées, sans serrage au départ, une dégradation du module pour des amplitudes de chargement croissantes, une accumulation de déformations irréversibles et des cycles ouverts montrant une certaine hystérésis.

Avec les modules de Young, les essais de compression permettent de mesurer les résistances en compression uniaxiale $\sigma_{\mathrm{c}}$. La Figure 9a montre l'histogramme des modules de Young mesurés sur les marnes en petites déformations (36 mesures). Une allure typique apparaît, d'une distribution 

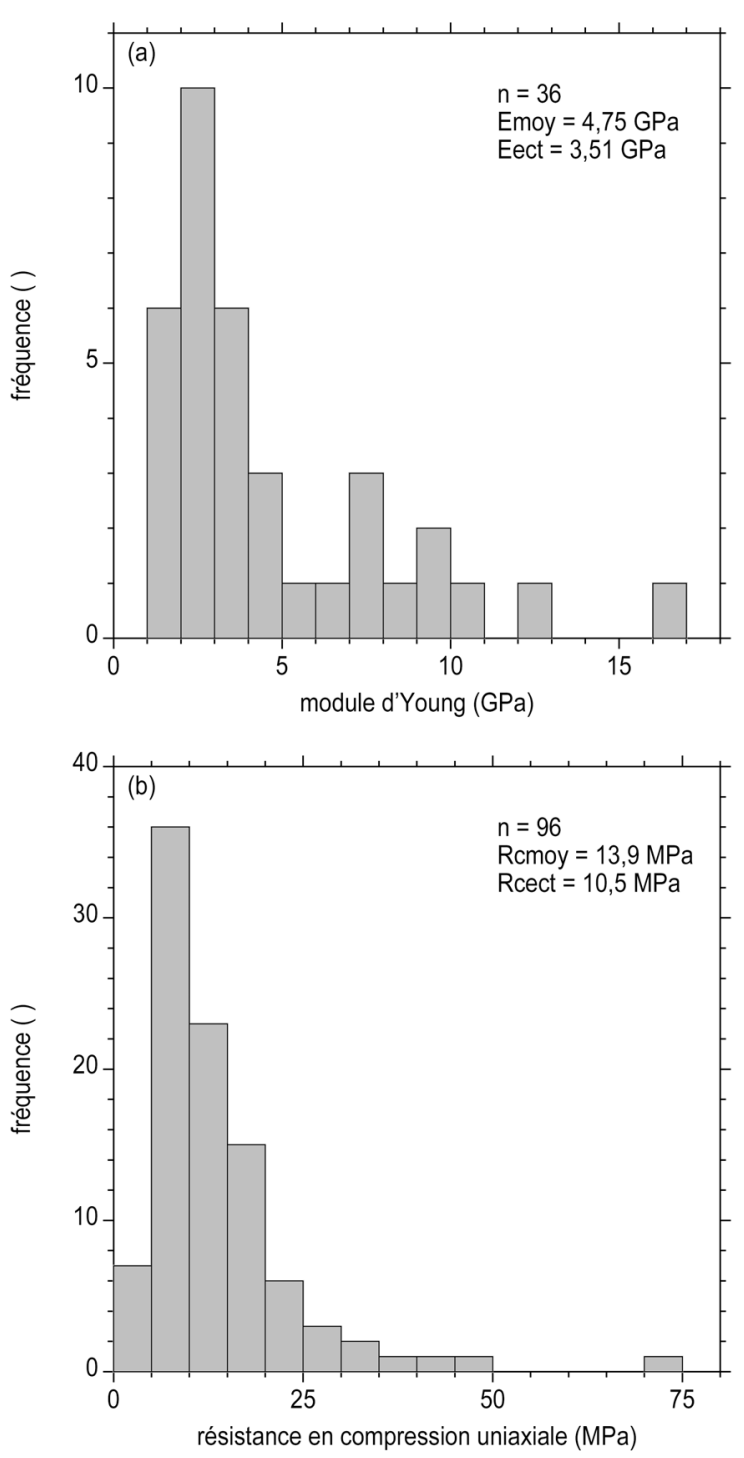

Fig. 9. Marnes indurées. a) Histogramme des modules de premier chargement en petites déformations. b) Histogramme des résistances en compression uniaxiale.

Fig. 9. Indurated marls. a) Histogram of first loading modulus at low strain. b) Histogram of unconfined compressive strength.

gamma qui est exprimée ici en échelles linéaires (distribution proche d'une distribution exponentielle). La moyenne des mesures est peu différente de leur écart-type $\left(E_{\mathrm{moy}}=4,75 \mathrm{GPa}\right.$ contre $E_{\text {ect }}=3,51 \mathrm{GPa}$ ). La Figure $9 \mathrm{~b}$ montre l'histogramme des résistances en compression uniaxiale avec une allure semblable (96 mesures, $\sigma_{\text {cmoy }}=13,9 \mathrm{MPa}, \sigma_{\mathrm{c} \text { ect }}=10,5 \mathrm{MPa}$ ). Les résistances les plus faibles sont mesurées en plus grand nombre, mais il n'est pas exclu de rencontrer une résistance très forte isolée (dans un niveau plus induré), ce qui est courant dans les massifs rocheux. Idem pour les modules.

\subsection{Anisotropie}

L'orientation du litage de la marne a été systématiquement relevée sur toutes les éprouvettes d'essais. Le graphique de la Figure 10a indique les mesures des modules de Young $E$ en fonction de l'angle $\alpha$ qui représente la direction des plans de litage par rapport à l'axe des éprouvettes. Pour ces essais, il n'a pas été possible de découper des éprouvettes dans toutes les directions à l'intérieur des échantillons carottés (diamètres des éprouvettes $50 \mathrm{~mm}$ et plus). Aussi, les données dépendent de l'orientation naturelle de la stratification par rapport à l'axe des sondages. Il apparaît sur ce graphique que le module de Young diminue lorsque $\alpha$ augmente. Des modules plus élevés sont obtenus pour un chargement dans une direction parallèle au litage. Des modules plus faibles sont obtenus pour un chargement dans une direction perpendiculaire au litage. Les modules les plus faibles sont obtenus pour un angle $\alpha$ égal à $50^{\circ}$ environ. Une interprétation sommaire de la variation des modules avec l'angle $\alpha$ est donnée sur la Figure 10a avec des courbes telles que (Jaeger, 1960; Donath, 1964; Liao et al., 1997 ; Nasseri et al., 2003) :

$$
E=A+B \cos (4 \alpha)+C \cos (2 \alpha)
$$

où $B=1500 \mathrm{MPa}, C=2000 \mathrm{MPa}$, puis $A=2500$ et $10000 \mathrm{MPa}$ pour les deux courbes extrêmes et $A=5000 \mathrm{MPa}$ pour la courbe moyenne ( $E$ en $\mathrm{MPa}$ et $\alpha$ en degrés). Cette interprétation conduit à un rapport d'anisotropie $E_{\alpha=0} / E_{\alpha=90}$ plutôt fort, égal à 1,9. En réalité, cette interprétation doit être pondérée par l'influence des caractéristiques d'état (teneur en eau et poids volumique), mais aussi par l'influence de la composition minéralogique de la marne (carbonates et argiles) et enfin par l'influence de la pression moyenne de confinement. Finalement, les données disponibles sont insuffisantes pour permettre une analyse plus fine de l'influence de ces paramètres, mais il peut être avancé que les marnes présentent une certaine anisotropie de déformabilité héritée de leur anisotropie de structure.

Les essais de résistance en compression uniaxiale et des essais de résistance en traction indirecte (brésiliens) aboutissent à des mesures très dispersées des résistances, en relation avec le mode de rupture de la marne, de type fragile, et qui est influencé par le litage (anisotropie de structure). Ainsi, suivant l'orientation de l'éprouvette par rapport au plan de litage, la rupture se produit sur l'un de ces plans, en travers de ces plans ou en relais sur plusieurs plans. De plus, la résistance dépend aussi de la teneur en carbonate de calcium, comme l'indique le graphique de la Figure 10b. Une interprétation de l'anisotropie de résistance est représentée sur la Figure 10c au moyen de relations de forme analogue à la précédente :

$$
\sigma_{c}=A+B \cos (4 \alpha)+C \cos (2 \alpha),
$$

avec $B=3 \mathrm{MPa}, C=-2,5 \mathrm{MPa}$, puis $A=4,5 \mathrm{MPa}$ et $19,5 \mathrm{MPa}$ pour les deux courbes extrêmes et $A=12 \mathrm{MPa}$ pour la courbe moyenne ( $E$ en $\mathrm{MPa}$ et $\alpha$ en degrés). Comme pour les modules, cette interprétation doit être pondérée par l'influence des caractéristiques d'état, mais aussi par l'influence de la composition minéralogique de la marne (carbonates et argiles). Toutefois, malgré la multiplication des essais, les données disponibles sont insuffisantes pour permettre une analyse plus fine, au-delà des tendances évoquées.

\section{Courbes d'état limite}

Plusieurs programmes expérimentaux ont été consacrés à la détermination de courbes d'état limite (CEL) de 

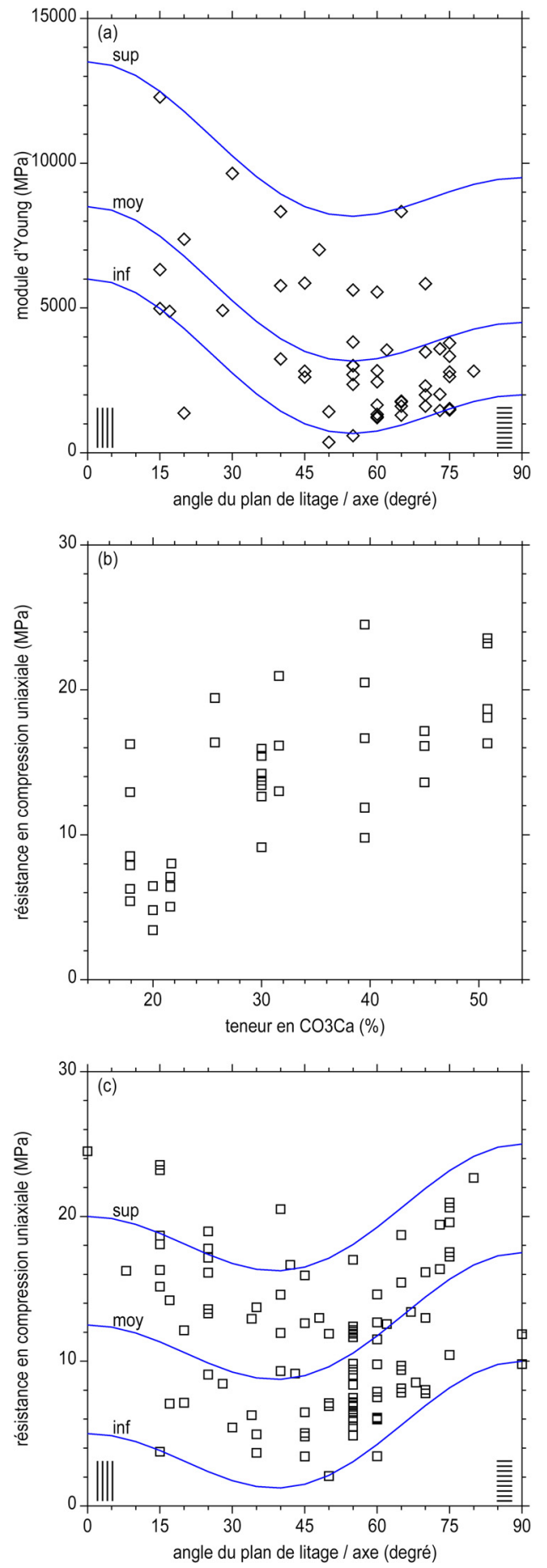

Fig. 10. Marnes indurées. a) Modules de déformation en compression uniaxiale en fonction de l'orientation des éprouvettes par rapport au litage. b) Résistances en compression uniaxiale en fonction de la teneur en carbonate de calcium. c) Résistances en compression uniaxiale en fonction de l'orientation des éprouvettes par rapport au litage.

Fig. 10. Indurated marls. a) Unconfined compression modulus versus angle of specimen axe relative to bedding plane. b) Unconfined compression strength versus content of calcium carbonate. c) Unconfined compression strength versus angle of specimen axe relative to bedding plane. marnes testées dans leur état naturel (Magnan et Serratrice, 1995; Serratrice, 1998, 2002). Deux autres exemples sont présentés ici.

\subsection{Marnes les plus tendres de l'Oligocène}

Des essais triaxiaux à haute pression et à chemin de contraintes contrôlé (chemin proportionnel ou radial) ont été réalisés sur des marnes argileuses de l'Oligocène décrites précédemment (famille 1, groupe 4; Tab. 1). Chaque éprouvette est soumise à un chargement continu drainé dans lequel la contrainte effective moyenne $p$ et le déviateur $q$ augmentent en maintenant constant le rapport $\eta=q / p$. Ce chargement proportionnel (ou radial) généralise le chargement oedométrique. Les essais sont réalisés à l'aide de machines triaxiales asservies à haute pression. Après une première phase de saturation (sous une contre-pression de $500 \mathrm{kPa}$ ) et de consolidation sous faible pression effective $\left(p_{\mathrm{c}}=100 \mathrm{kPa}\right)$, le déviateur $q$ et la pression moyenne effective $p$ sont augmentés proportionnellement dans un rapport $\eta=\Delta q / \Delta p$ donné, vers les hautes pressions, à vitesse lente, en condition drainée. Les déformations axiales $\varepsilon_{a}$ et volumiques $\varepsilon_{v}$ sont enregistrées pendant le chargement. Des cycles de chargement-déchargement peuvent être envisagés comme à l'oedomètre. En fin de chargement radial, l'essai est poursuivi par une phase de cisaillement drainé ou non drainé en compression $(\Delta q>0)$ ou en extension $(\Delta q<0)$.

Dans le cadre de ce programme expérimental, 13 éprouvettes ont été testées ainsi. Les pentes $\eta$ ont été choisies entre $-0,64$ et 0,75 . La durée du chargement va de 74 à 167 heures environ, pour des pressions moyennes maximales de l'ordre de $10 \mathrm{MPa}$ (soit des vitesses de chargement de l'ordre de $1 \mathrm{kPa} / \mathrm{min}$ ). Un essai a fait l'objet d'un cycle de chargementdéchargement (figuré « $Y »)$. Les graphiques des Figures 11a à $11 \mathrm{~d}$ montrent des réponses enregistrées pendant une partie des 13 essais radiaux réalisés. Il s'agit des courbes contraintesdéformations $\left(\varepsilon_{\mathrm{a}}, q\right)$ et $\left(q, \varepsilon_{\mathrm{v}}\right)$, des chemins contrôlés en contraintes effectives $(p, q)$ et des chemins de déformation $\left(\varepsilon_{\mathrm{a}}, \varepsilon_{\mathrm{v}}\right)$.

Le graphique de la Figure 11e montre les courbes contraintes-déformations «volumiques» en échelles semilogarithmiques $\left(\varepsilon_{\mathrm{v}}, \lg p\right)$. Ces dernières s'apparentent aux courbes de compressibilité oedométrique des argiles surconsolidées, qui sont caractérisées par deux segments de droites se raccordant par un coude au point d'état limite de coordonnées $\left(\lg p_{\mathrm{p}}, e_{\mathrm{p}}\right)$. En fait, les courbes $\left(\varepsilon_{\mathrm{v}}, \lg p\right)$ sont progressives et ne présentent pas des coudes marqués. Elles font apparaître deux régions néanmoins, une région pseudoélastique au début du chargement avec une forte rigidité, puis une région de déformation plastique à hautes pressions. Chacune de ces régions est caractérisée par une droite dont un encadrement est proposé sur la Figure 11e avec des pentes $d l g p / d \varepsilon_{\mathrm{v}}$ égales à 56,4 et 10,8 respectivement. Ces valeurs semblent à peu près constantes et indépendantes de la pente $\eta$ des chemins proportionnels. Les inverses de ces pentes sont $C^{*}{ }_{s}=1 / 56,4=0,018$ et $C^{*}{ }_{\mathrm{c}}=1 / 10,8=0,092$. Le rapport de ces pentes vaut $56,4 / 10,8=5,2$. Exprimées en indices des vides $e$ au moyen de la relation $e=e_{0}-\left(1+e_{0}\right) \varepsilon_{\mathrm{v}}$ où $e_{0}=0,40$, les deux branches des courbes de compressibilité $(\lg p, e)$ des marnes argileuses ont pour pentes $C_{\mathrm{s}}=0,025$ et $C_{\mathrm{c}}=0,13$. 
Entre ces deux régions extrêmes des courbes contraintesdéformations $\left(\varepsilon_{\mathrm{v}}, \lg p\right)$, le chargement impose à la marne de franchir la courbe d'état limite (CEL) qui sépare son domaine pseudo-élastique de son domaine plastique. En principe, le point de plastification peut être défini comme à l'oedomètre par les coordonnées du point d'intersection des droites de pentes $C_{s}$ et $C_{c}$ pour chacun des chemins proportionnels. Les coordonnées $\left(\varepsilon_{v p}, q_{p}\right)$ ainsi pointées sur les courbes $\left(\varepsilon_{v}, \lg p\right)$ sont associées aux points $\left(p_{p}, q_{p}\right)$ du chemin des contraintes. Ces états de contraintes de pré-consolidation dépendent de la pente $\eta$. Ainsi, les coudes des courbes de compressibilité des marnes argileuses existent pour chacun des chemins des contraintes proportionnels. Mais ils sont en partie estompés par la forte rigidité de la marne et en raison de la faible différence entre le coefficient de gonflement $C_{s}$ à faible pression (domaine pseudo-élastique) et le coefficient de compressibilité $C_{c}$ à haute pression (domaine plastique). Ainsi, la faible courbure des courbes $\left(\varepsilon_{v}, \lg p\right)$ rend malaisée la détermination des seuils d'état limite. Dans ces conditions, et compte tenu de la variabilité des propriétés de la marne, la précision des mesures de déformation ne permet pas d'identifier clairement ces coudes.

Aussi, différentes méthodes ont été recherchées pour déterminer les coordonnées de ces points le long de chacun des chemins radiaux. Les chemins des déformations font apparaître un changement de direction à l'arrivée dans les hautes pressions. Une analyse précise de ces chemins a permis de retenir deux points particuliers représentant l'amorce de la courbure d'une part, puis le passage à un régime plastique à haute pression d'autre part. Les coordonnées $\left(p_{e}, q_{e}\right)$ et $\left(p_{p}, q_{p}\right)$ de ces points le long des chemins radiaux permettent de prédéterminer deux courbes d'état limite emboîtées, notées $\mathrm{CEL}^{e}$ et $\mathrm{CEL}^{p}$, dans le plan des contraintes effectives $(p, q)$ de la Figure 11b. Ces courbes délimitent un domaine pseudoélastique à l'intérieur de la $\mathrm{CEL}^{e}$ où les déformations élastiques sont dominantes, un domaine plastique à l'extérieur de la courbe $\mathrm{CEL}^{p}$ où les déformations plastiques sont dominantes et un domaine intermédiaire compris entre ces deux courbes. L'enveloppe $\mathrm{CEL}^{p}$ est encadrée par les deux droites de Coulomb qui représentent la résistance de la marne dans son domaine plastique, de pente $M_{c}=0,86$ et $M_{e}=-0,67$ (Fig. 4 et Tab. 2). L'ensemble de ces points constitue la « face isotrope » de la courbe d'état limite dans le plan des contraintes effectives $(p, q)$.

En associant les points d'état limite $\left(p_{p}, q_{p}\right)$ des chemins radiaux avec les pics de résistance en compression et en extension obtenus en cisaillement à basse pression et exprimés par les couples de résistance $\left(p_{r}, q_{r}\right)$ d'après le critère $(q / p)_{\max }$, il est possible de trouver les courbes d'état limite de la forme suivante dans le plan $(p, q)$ (Magnan et Serratrice, 1995):

$$
\begin{aligned}
p= & p_{o}\left[\eta_{d 1}^{2} \exp \left(-\left(\eta-\eta_{1}\right)^{2} / \eta_{d 1}^{2}\right)\right. \\
& \left.+\eta_{d 2}{ }^{2} \exp \left(-\left(\eta-\eta_{2}\right)^{2} / \eta_{d 2}^{2}\right)\right] /\left(\eta_{d 1}^{2}+\eta_{d 2}{ }^{2}\right),
\end{aligned}
$$

avec cinq paramètres $p_{\mathrm{o}}, \eta_{1}, \eta_{d 1}, \eta_{2}$ et $\eta_{d 2}$. L'identification des paramètres s'effectue dans le plan $(\eta, \lg p)$ en échelles semilogarithmiques, où $\eta=q / p$. Les paramètres retenus pour caractériser ces enveloppes sont indiquées dans le Tableau 2. Avec ce choix, seul $p_{0}$ change pour distinguer les deux enveloppes, qui se trouvent ainsi homothétiques dans le plan des contraintes effectives. Elles ont été représentées sur la Figure 11b. Par la suite, une étape supplémentaire a consisté à déterminer les déformations associées aux courbes $\mathrm{CEL}^{e}$ et $\mathrm{CEL}^{p}$, puis les incréments de déformations associés aux incréments de contraintes le long des chemins radiaux. Les directions des vecteurs incréments de contraintes et de déformations de coordonnées $\Delta p, \Delta q, \Delta \varepsilon_{a}$, et $\Delta \varepsilon_{v}$ sont indiquées sur les graphiques de la Figure 11 en accompagnement de la trace de la $\mathrm{CEL}^{p}$ dans les plans des contraintes effectives et des déformations.

\subsection{Marne de Ceyras}

Cet autre exemple concerne une marne homogène grise argilo-silteuse du Miocène moyen qui a été prélevée à Ceyras dans le département de l'Hérault. La marne grise a été recueillie au moyen de deux sondages carottés dans un talus subvertical, l'un vertical et l'autre horizontal. La marne possède un indice des vides moyen égal à 0,48 .

Des essais triaxiaux ont été réalisés. Les Figures $12 \mathrm{a}$ et $\mathrm{b}$ montrent les chemins des contraintes effectives $(p, q)$ recueillis pendant les chemins radiaux puis les phases de cisaillement non drainé à haute pression qui font suite, pour les éprouvettes d'axe vertical et les éprouvettes d'axe horizontal respectivement. À haute pression, les chemins des contraintes suivis pendant les cisaillements non drainés sont incurvés vers les pressions effectives moyennes décroissantes, ce qui traduit un chargement de la marne dans son domaine plastique. À basse pression, les chemins des contraintes témoignent au contraire du caractère surconsolidé puis dilatant de la marne. Les points à la rupture $\left(p_{r}, q_{r}\right)$ sont définis par le critère maximal $|q / p|_{\text {max }}$. Dans le domaine plastique de la marne sous les fortes pressions, la rupture s'établit sur des droites de Coulomb de compression et d'extension qui passent par l'origine du plan des contraintes effectives $(p, q)$ et pour lesquelles la cohésion est nulle. Ces droites de Coulomb ont pour pentes $M_{c}=1,25$ en compression et $M_{e}=-0,88$ en extension, soit $c^{\prime}=0$ et $\varphi^{\prime}=31,1$ $\circ$, indépendamment de la direction du prélèvement de la marne. À basse pression, l'effet de structure se signale par un supplément de résistance qui se manifeste par une enveloppe de rupture courbe.

Par contre et malgré le nombre limité d'essais et la dispersion des résultats, les courbes d'état limite ne coïncident pas, comme le montrent les Figures $12 \mathrm{a}$ et b. Il semble nécessaire de distinguer les échantillons verticaux et horizontaux. Les premiers possèderaient une CEL plus étendue du côté des compressions, formant un «nez» centré sur un chemin oedométrique. Au contraire, la CEL des éprouvettes d'axes horizontaux semble plus arrondie et centrée sur l'axe isotrope. Le Tableau 3 récapitule les paramètres identifiés dans chacune des deux directions de prélèvement, selon l'équation (7). À la rupture dans le domaine normalement consolidé à haute pression, cette anisotropie semble s'effacer (pentes identiques $M_{c}$ et $M_{e}$ pour les deux directions de prélèvement). Ce comportement est analogue à celui déjà observé avec la marne du site de Bompas (Fig. 7). La pression $p_{0}=8,2 \mathrm{MPa}$ témoigne du degré d'induration de la marne, qui pourtant, possède l'indice des vides le plus grand des matériaux examinés ici. 


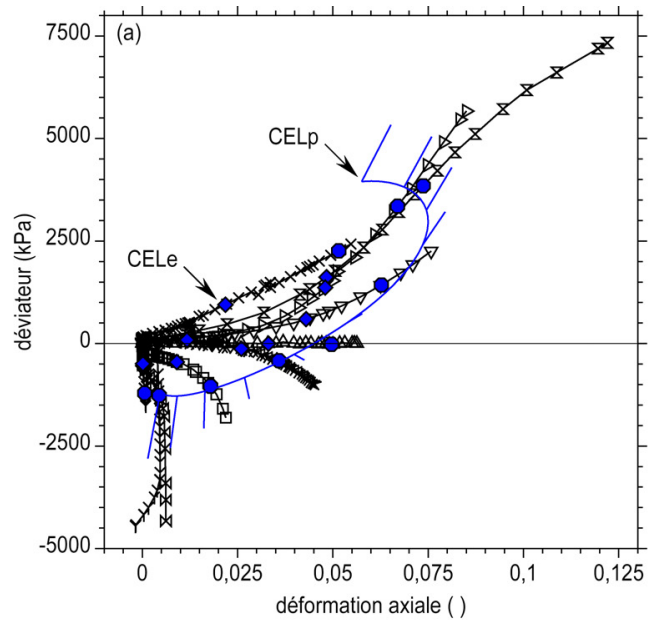

+ radial éta $=0,00$ $\times$ radial éta $=0,39 \quad \boldsymbol{z}$ radial éta $=0,75$

$\square$ radial éta $=-0,30 \quad \square$ radial éta $=0,57$

$\triangle$ radial éta $=0,00 \quad$ y radial éta $=-0,64$

$\nabla$ radial éta $=0,25 \quad *$ radial éta $=-0,10$

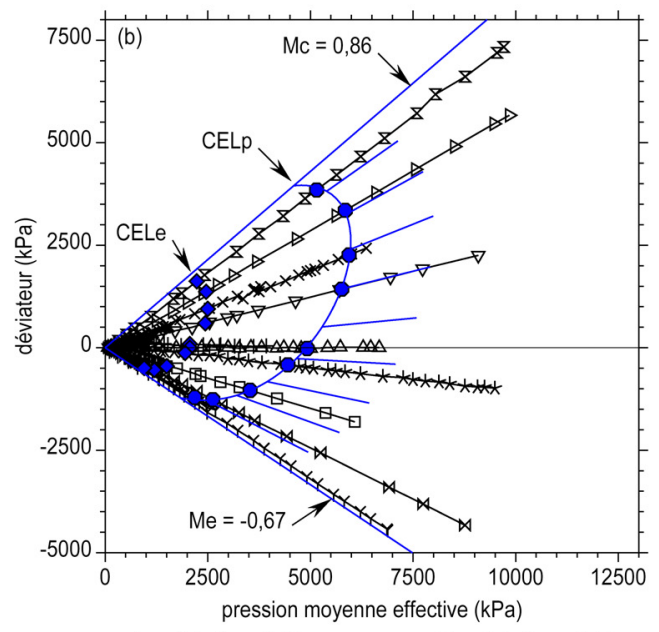

+ radial éta $=0,00$

$\times$ radial éta $=0,39$

$\square$ radial éta $=-0,30$

$\triangle$ radial éta $=0,00$

$\nabla$ radial éta $=0,25$

$\rtimes$ radial éta $=-0,49$

$\varangle$ radial éta $=0,75$

$\triangle$ radial éta $=0,57$

Y radial éta $=-0,64$

$\star$ radial éta $=-0,10$

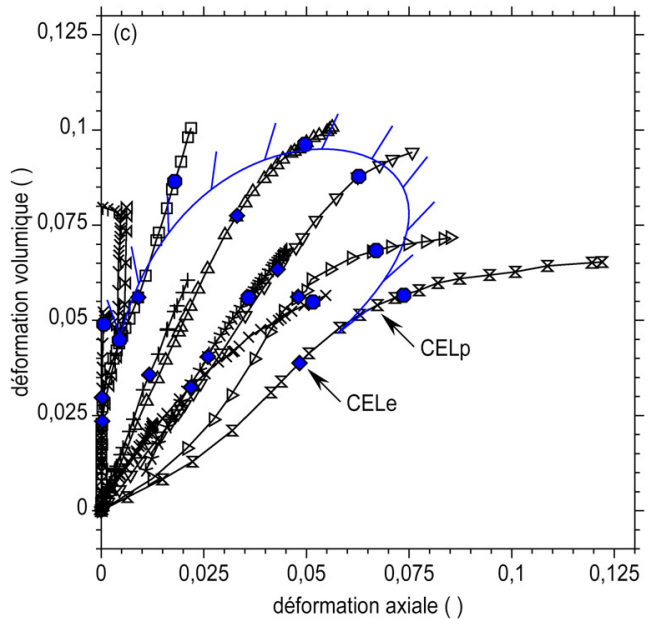

+ radial éta $=0,00$ $\times$ radial éta $=0,39$ $\square$ radial éta $=-0,30$

$\Delta$ radial éta $=0,00$

$\nabla$ radial éta $=0,25$ $\bowtie$ radial éta $=-0,49$ $\varangle$ radial éta $=0,75$ $\triangle$ radial éta $=0,57$

$Y$ radial éta $=-0,64$

$\star$ radial éta $=-0,10$

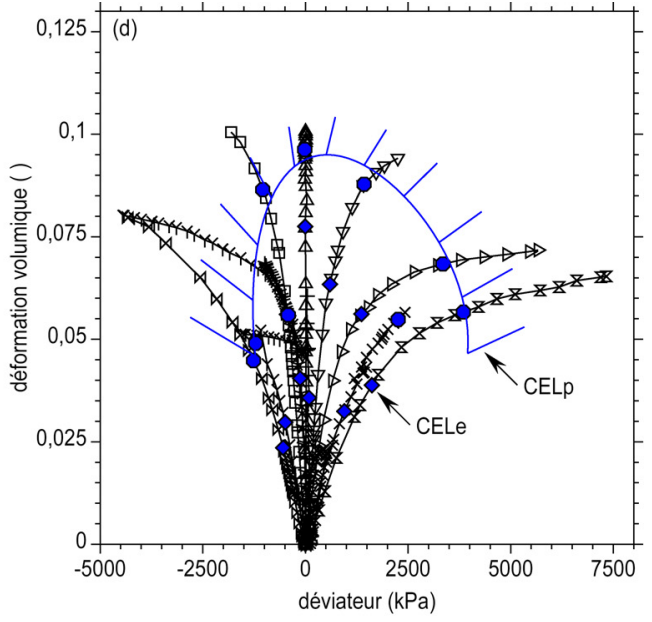

+ radial éta $=0,00$

$\times$ radial éta $=0,39$

$\square$ radial éta $=-0,30$

$\triangle$ radial éta $=0,00$

$\triangle$ radial éta $=-0,49$

$\mathbf{z}$ radial éta $=0,75$

$\nabla$ radial éta $=0,25$

Y radial éta $=-0,64$

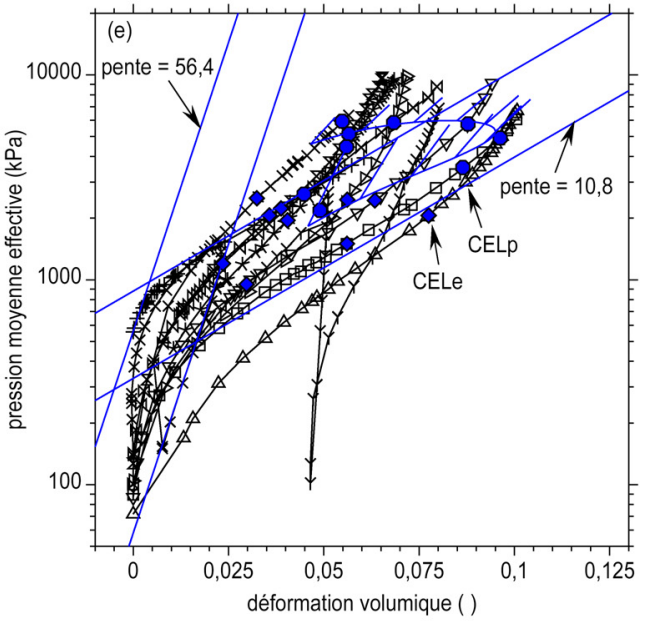

+ radial éta $=0,00$

$\times$ radial éta $=0,39$

$\square$ radial éta $=-0,30$

$\Delta$ radial éta $=0,00$

$\bowtie$ radial éta $=-0,49$

$\triangle$ radial éta $=0,75$

$\triangle$ radial éta $=0,57$

$Y$ radial éta $=-0,64$

$\nabla$ radial éta $=0,25$

Fig. 11. Essais triaxiaux à chemins contrôlés radiaux. a) Courbes contrainte-déformation $\left(\varepsilon_{\mathrm{a}}, q\right)$. b) Chemins des contraintes effectives $(p, q)$. c) Chemins des déformations $\left(\varepsilon_{a}, \varepsilon_{v}\right)$. d) Déformations volumiques déviateur $\left(q, \varepsilon_{v}\right)$. e) Courbes contraintedéformation $\left(\varepsilon_{\mathrm{v}}, \operatorname{lgp}\right)$.

Fig. 11. Radial controlled stress path triaxial tests. a) Stress-strain curves $\left(\varepsilon_{a}, q\right)$. b) Effective stress paths $(p, q)$. c) Strain paths. d) Volumetric strain versus deviator stress. e) Stress-strain curves $\left(\varepsilon_{v}, \lg p\right)$. 
Tableau 2. Caractéristiques des CEL des marnes argileuses de l'Oligocène.

Table 2. CEL parameters of Oligocene clayed marls.

\begin{tabular}{llllll}
\hline CourbeCEL & $p_{\mathrm{o}}(\mathrm{kPa})$ & $\begin{array}{l}\eta_{1} \\
(-)\end{array}$ & $\begin{array}{l}\eta_{d 1} \\
(-)\end{array}$ & $\begin{array}{l}\eta_{2} \\
(-)\end{array}$ & $\begin{array}{l}\eta_{d 2} \\
(-)\end{array}$ \\
\hline élastique & 2800 & 0,20 & 0,90 & 0,70 & 0,60 \\
plastique & 6650 & 0,20 & 0,90 & 0,70 & 0,60 \\
\hline
\end{tabular}

\section{Marnes fissurées, marnes altérées}

Les massifs marneux portent souvent les traces de mouvements tectoniques qui ont affecté leur histoire géologique. Les terrains sont alors fracturés et altérés en profondeur. Leurs propriétés mécaniques s'en trouvent fragilisées. Dans d'autres situations, les régions superficielles des massifs marneux sont altérées avec, là aussi, une dégradation de leurs propriétés mécaniques. Ces processus de fissuration et d'altération mettent en défaut l'apport des effets de structure en matière de comportement mécanique des marnes, ce qui pousse à développer des méthodes efficaces de caractérisation de ces terrains pour les besoins des projets concernés.

\subsection{Marnes fissurées}

La résistance des marnes fissurées doit être mesurée au laboratoire dans diverses situations où des projets concernent des pentes naturelles, des talus de déblai, des excavations ou des tunnels. Le prélèvement de ces matériaux dans leur état naturel au cœur des massifs présente de réelles difficultés si bien que des adaptations des procédures d'essais sont nécessaires suivant que le mode de prélèvement est effectué par carottage ou par blocs et suivant la quantité d'échantillons disponibles. Ces terrains fissurés sont souvent très hétérogènes et leur déformabilité et leur résistance sont très dispersées. Ils sont sensibles au remaniement. Aussi, il est recommandé de multiplier le nombre d'essais afin de mieux qualifier la résistance au cisaillement de ces terrains. Souvent, il est difficile, voire impossible, de maîtriser l'orientation des plans de discontinuité pendant les essais, que ces plans soient visibles initialement, ou pas, en périphérie des échantillons disponibles. De plus, car ils sont prélevés en profondeur, les terrains sont saturés et ils doivent être testés dans cet état et dans des gammes de pressions étendues. Pour ces différentes raisons, l'essai triaxial présente un réel intérêt en matière de mesure des résistances des marnes fissurées.

L'exemple présenté ici concerne les marnes prélevées par carottage dans la région de Besançon (site du Bois du Peu). Ces marnes possèdent des propriétés très dispersées. Elles sont compactes avec un indice des vides moyen égal à 0,29 . Des essais triaxiaux drainés et non drainés ont été réalisées sur des éprouvettes découpées dans les échantillons carottés et testées sous des pressions effectives de consolidation comprises entre 50 et $2000 \mathrm{kPa}$. Les courbes contraintes déformation $\left(\varepsilon_{a}, q\right)$ sont représentées sur la Figure 13a. Ces courbes se singularisent par une absence de pic quelque soient les pressions de confinement. Les chemins des contraintes effectives sont donnés sur la Figure 13b. La dispersion des résistances est très forte. L'effet de la fissuration est indépendant des pressions de confinement. Néanmoins, les
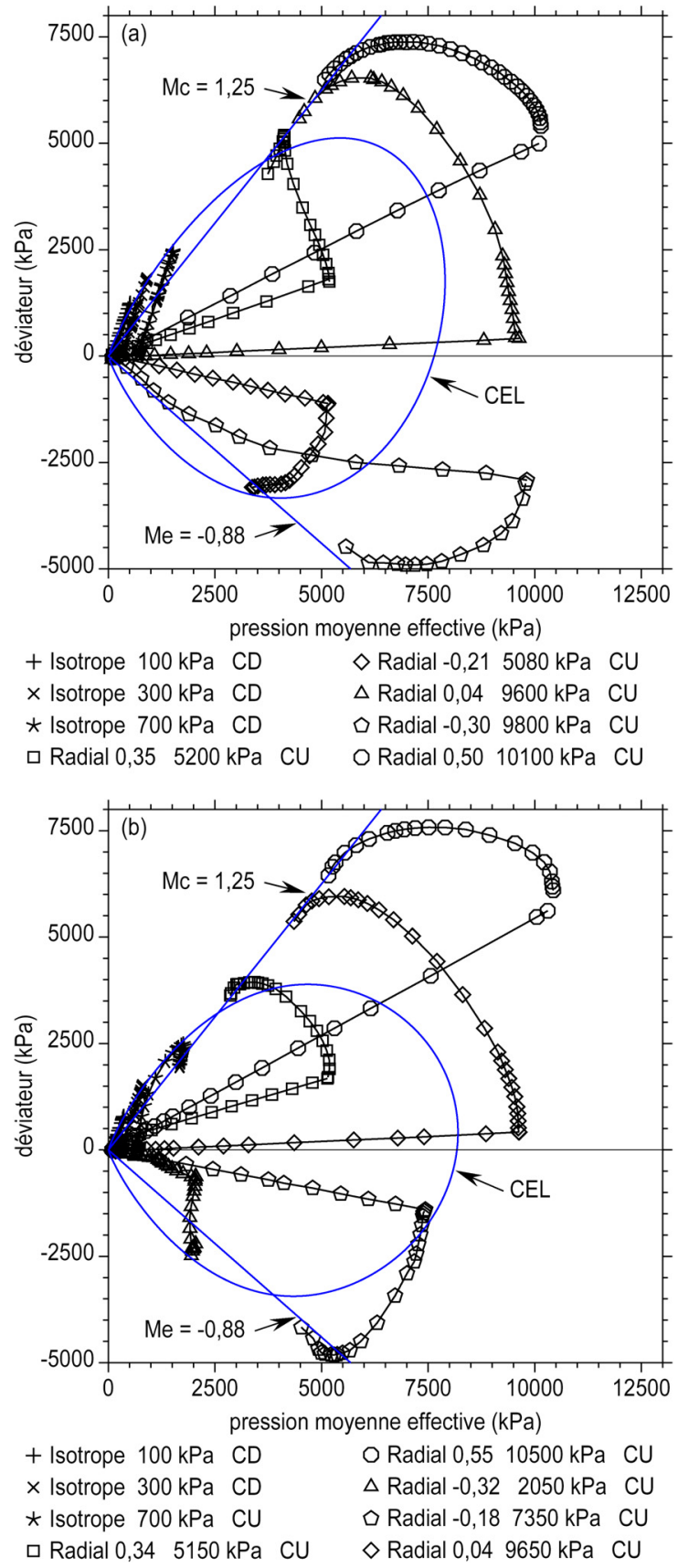

Fig. 12. Comportement mécanique de la marne grise. Essais triaxiaux drainés à chemins radiaux et cisaillement non drainé à haute pression CU HP. Plans des contraintes effectives (p, q). a) Sondage vertical. b) Sondage horizontal.

Fig. 12. Mechanical behaviour of grey marl. Radial controlled stress path loading and undrained shearing at high pressure $C U H P$. Effective stress plane $(p, q)$. a) Vertical borehole. b) Horizontal borehole.

marnes les plus compactes sont les plus résistantes. Les points à la rupture doivent être encadrés par deux enveloppes de rupture courbes qui sont définies au moyen d'une loi puissance :

$$
q_{r}=a_{5}\left(p_{r} / p_{u}\right)^{\zeta}
$$


Tableau 3. Caractéristiques des CEL de la marne de Ceyras. Table 3. CEL parameters of Ceyras marl.

\begin{tabular}{llllll}
\hline Sondage & $p_{\mathrm{o}}(\mathrm{kPa})$ & $\begin{array}{l}\eta_{1} \\
(-)\end{array}$ & $\begin{array}{l}\eta_{d 1} \\
(-)\end{array}$ & $\begin{array}{l}\eta_{2} \\
(-)\end{array}$ & $\begin{array}{l}\eta_{d 2} \\
(-)\end{array}$ \\
\hline vertical & 8200 & 0 & 1,3 & 0,45 & 1,0 \\
horizontal & 8200 & 0,05 & 0,5 & 0,05 & 1,2 \\
\hline
\end{tabular}
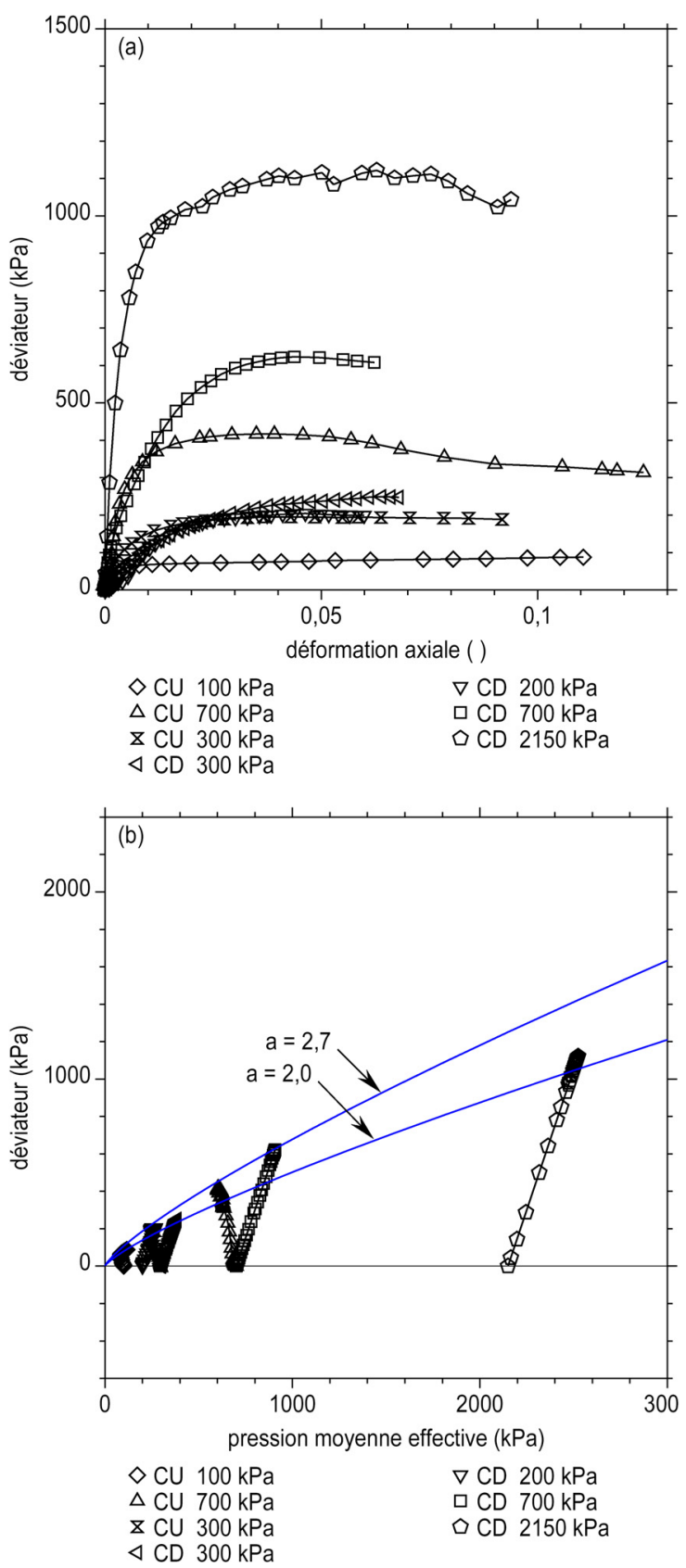

Fig. 13. Marnes fissurées. Essais triaxiaux $\mathrm{CD}$ et $\mathrm{CU}$ à moyenne pression. a) Courbes contrainte-déformation. b) Chemins des contraintes effectives.

Fig. 13. Fissured marls. Medium pressure $C D$ and $C U$ triaxial tests. a) Stress-strain curves. b) Effective stress paths. où $\zeta=0,8$ ici et $a_{5}=2,0 \mathrm{kPa}$ et $2,7 \mathrm{kPa}$, avec $p_{r}$ et $q_{r}$ en $\mathrm{kPa}$ et $p_{u}$ la pression unité $\left(p_{u}=1 \mathrm{kPa}\right)$. Une réponse analogue a été observée avec les marnes du Toarcien de la région de Millau (Serratrice, 2013). La tangente à cette enveloppe de rupture définie par l'équation (8) pour $p_{r}=300 \mathrm{kPa}$ a pour caractéristiques de résistance au cisaillement $c^{\prime}=18 \mathrm{kPa}$ et $\varphi^{\prime}=13,6^{\circ}$.

Dans les marnes fissurées ou schisteuses les mesures des résistances le long de discontinuités montrent que les discontinuités exprimées ou latentes guident la rupture des éprouvettes triaxiales. Le plus souvent, les cisaillements s'effectuent sans pic ou des pics peu marqués. Cette observation est vraie dans le cas des fissures préexistantes, mais aussi pour les fissures déclarées pendant le cisaillement triaxial et dont l'orientation est indépendante de la sollicitation imposée. Les hautes pressions ne changent pas cette tendance et la cinématique de rupture est toujours guidée par les fissures naturelles. Dans tous les cas, il est préférable d'interpréter les essais triaxiaux en contrainte normale et en contrainte de cisaillement exercée sur les plans de rupture principaux. Les angles de frottement ainsi identifiés sont plus petits que les angles obtenus par une interprétation conventionnelle des essais triaxiaux. Ces angles sont souvent très faibles et représentatifs des angles de frottement résiduels. Les enveloppes de rupture montrent une faible courbure, indiquant une légère évolution de la résistance avec la pression moyenne. Toutefois, la dispersion des résistances occulte souvent cette tendance quand le nombre d'essais est limité.

\subsection{Marnes altérées}

Des sondages carottés ont permis de prélever des marnes de l'Aptien dans la région de St Saturnin les Apt (Vaucluse). L'un des sondages a permis de prélever une marne relativement tendre en place sous un horizon altéré épais. Cette opportunité a été mise à profit en effectuant des essais d'identification et des essais triaxiaux à haute pression en vue de comparer les propriétés de la marne à celles de l'argile d'altération (Serratrice et al., 2012). Les caractéristiques d'état des matériaux sont obtenues à partir des éprouvettes d'essais découpées manuellement au sein des échantillons carottés et à partir de fragments de ces échantillons. Les mesures des teneurs en eau et des poids volumiques montrent des distributions bi-modales qui séparent les deux familles de terrain correspondant aux argiles superficielles et aux marnes. Les indices des vides sont égaux respectivement à 0,60 et 0,39 en moyenne.

Le programme expérimental a donné l'occasion de comparer la résistance de la marne intacte à celle de son équivalent altéré. Pour cela, des essais triaxiaux à haute pression ont été mis en œuvre sur les deux matériaux, avec des pressions effectives de consolidation atteignant $7000 \mathrm{kPa}$ pour la marne et $4000 \mathrm{kPa}$ pour l'argile. Le graphique de la Figure 14 montre les points à la rupture mesurées et reportées dans le plan des contraintes effectives $(p, q)$. Deux paraboles permettent d'expliquer les résistances mesurées dans la gamme des pressions exercées. Les équations de ces lois puissances sont de la forme (8) où $\zeta=0,73$ et $a_{5}=6 \mathrm{kPa}$ et $a_{5}=11 \mathrm{kPa}$, avec $p_{r}$ et $q_{r}$ en kilopascals. Les deux expressions possèdent le même exposant. La résistance des marnes est à 


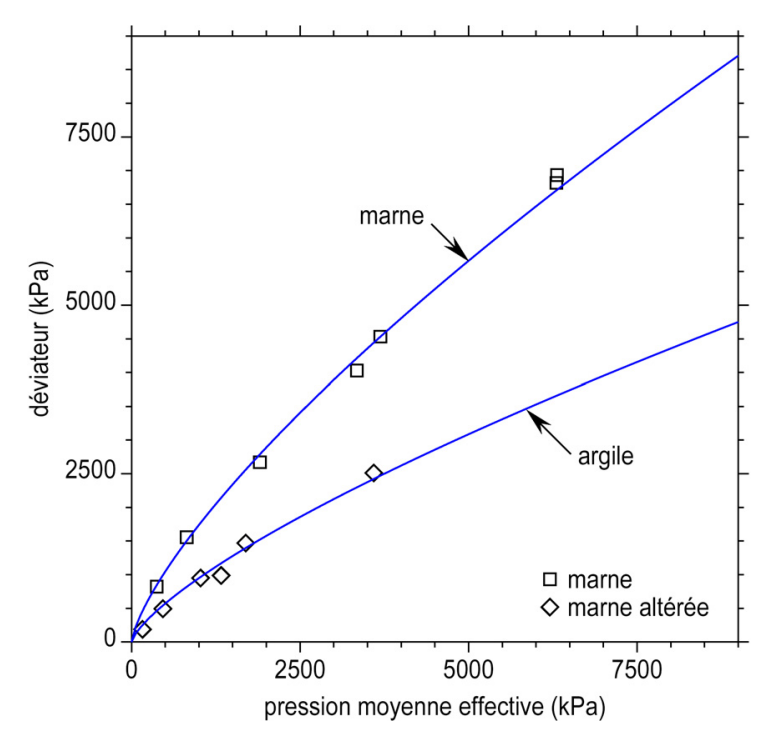

Fig. 14. Marne intacte et marne altérée. Enveloppes de rupture. Points expérimentaux et enveloppes en lois puissances.

Fig. 14. Intact marl and weathered marl. Failure envelopes. Experimental data and power law envelopes.

peu près deux fois plus grande que celle des argiles d'altération. Les marnes présentent un comportement fragile très dilatant à basse pression, contrairement aux argiles. Les essais CD et CU conduisent aux mêmes enveloppes de rupture, à la dispersion près des mesures.

\section{Conclusion}

Des exemples ont été présentés pour illustrer la diversité des comportements mécaniques des marnes d'après les essais de laboratoire. Ces terrains présentent une grande variété lithologique et d'états et sont largement rencontrés dans différentes régions en France et dans le monde. Ces données ont été recueillies au fil du temps à l'occasion des reconnaissances géotechniques de sites réalisées dans le cadre de projets d'ouvrages de génie civil et des projets d'ouvrages souterrains en particulier. Tous ces matériaux sont testés dans leur état naturel.

Peu d'études expérimentales ont été consacrées au comportement mécanique des marnes au laboratoire. L'accent a été porté sur cet aspect ici. Mais, il ne faut pas perdre de vue l'importance des propriétés physiques et minéralogiques de ces matériaux en accompagnement de leurs caractéristiques mécaniques, à commencer par la compacité. Dans les exemples présentés, les indices des vides des marnes testées sont tous inférieurs à 0,5 . Les données expérimentales ont été recueillies au moyen d'essais triaxiaux en appliquant différents types de chargements isotropes, proportionnels ou des cisaillements en compression ou en extension. L'utilisation de machines triaxiales à haute pression est rendue nécessaire par les propriétés des terrains qui sont peu compressibles et résistants en comparaison des argiles. Ces essais permettent d'établir une analogie entre le comportement des marnes observé à haute pression et celui des argiles à basse pression. Pour les marnes homogènes, cette analogie témoigne des effets de structures présents dans les marnes.
Les marnes homogènes et plus ou moins raides présentent des modules de Young élevés et intermédiaires entre les argiles et les roches dures, en fonction du niveau d'induration. Ils augmentent avec la pression moyenne. Ces marnes homogènes présentent une anisotropie marquée en petites déformations quand elles possèdent une anisotropie de structure bien exprimée. Cette anisotropie se manifeste aussi à la rupture à basse pression, mais elle s'estompe à haute pression. Sinon, dans la majorité des cas, les marnes homogènes ne sont pas ou sont faiblement anisotropes. À basse pression, les enveloppes de rupture sont courbes en compression et en extension triaxiale. Pour les marnes les plus tendres, ces enveloppes se prolongent par des droites de Coulomb sans cohésion dans le domaine plastique à haute pression. Pour les marnes plus indurées, les enveloppes de rupture courbes se prolongent à haute pression sans changement d'allure, à l'instar des roches.

De même et au moyen de chargements proportionnels, il est possible d'identifier une courbe d'état limite pour les marnes les plus tendres. Cette courbe constitue une frontière côté isotrope du plan des contraintes effectives entre le domaine pseudo-élastique et le domaine plastique du matériau. Des déformations volumiques significatives et irréversibles prennent naissance au franchissement de cette frontière relativement étroite. Les marnes indurées présentent des courbes d'état limite emboîtées qui gagnent rapidement en étendue avec l'induration. Dans ce cas, l'identification de cette courbe d'état limite n'est plus accessible à l'expérience dans la gamme de pressions de l'ordre de la dizaine de mégapascals. Cette frontière paraît d'autant moins bien exprimée que les matériaux sont indurés et très peu compressibles. Ainsi, par opposition avec les argiles sensibles et les roches poreuses, les marnes apparaissent comme des matériaux «pleins» (c'est-à-dire faiblement poreux), peu déformables sous les sollicitations à tendance isotrope. Il s'agit là d'une expression particulière des effets de structure qui caractérisent le comportement mécanique des marnes.

À l'inverse, la fissuration et l'altération constituent des facteurs de réduction des effets de structure qui s'accompagnent par une dégradation des propriétés mécaniques initiales. Les sites où les marnes se rencontrent à des degrés divers de fissuration et d'altération sont les plus nombreux. De fait, l'étude des marnes fissurées et altérées présente un intérêt pratique très important. Ici, des méthodes spécifiques ont été mises en œuvre sur la base d'essais triaxiaux pour étudier les marnes fissurées dans des contextes où, souvent, le prélèvement sur site constitue un premier écueil d'importance qui limite la quantité et la qualité des matériaux disponibles à tester au laboratoire. Ces facteurs de dégradation jouent avec une certaine progressivité à l'opposé des effets de structure, ce qui conduit à une grande diversité d'états au sein des formations géologiques concernées. De ce fait, les propriétés mécaniques sont mesurées avec une large dispersion, contre laquelle la multiplication des essais trouve rapidement ses limites.

Enfin, les marnes constituent un groupe particulier parmi les sols indurés et les roches tendres. Elles présentent un large spectre de comportements et de propriétés mécaniques en liaison avec leur diversité lithologique et d'états et en écho aux processus contradictoires qui favorisent les effets de structure ou les effets opposés de dégradation de leurs caractéristiques. Les résultats expérimentaux présentés 
ici concordent avec des observations similaires publiées dans la littérature. Au travers de leur comportement mécanique, les marnes portent la plupart des indices qui traduisent les effets de structure inventoriés dans les sols surconsolidés et les autres types de sols indurés et de roches tendres. Une meilleure connaissance de ces comportements trouve ses applications dans les projets d'ouvrages et principalement dans les projets des ouvrages souterrains et des excavations.

\section{Références}

Alonso EE, Pineda JA, Cardoso R. 2010. Degradation of marls: two case studies from the Iberian Peninsula. Eng Geol Special Pub 23: $47-75$.

Amorosi A, Rampello S. 2007. An experimental investigation into the mechanical behaviour of a structured stiff clay. Géotechnique 57 (2): 153-166.

Anagnostopoulos AG, Kalteziotis N, Tsiambaos GK, Kavvadas M. 1991. Geotechnical properties of the Corinth Canal marls. Geotech Geol Eng 9(1): 1-26.

Aversa S, Evangelista A, Leroueil S, Picarelli L. 1993. Some aspects of the mechanical behaviour of 'structured' soils and soft rocks. In: Proceedings of the International Symposium on Geotechnical Engineering of Hard Soils and Soft Rocks, Athens, Vol. 1, pp. 359366.

Bekkouche A, Djeddid A, Aissa Mamoune SM. 2002. Identification et prévision du gonflement des marnes de Tlemcen (Algérie). In: Actes du Symposium International PARAM 2002, Paris, pp. 125-132.

Biarez J, Hicher PY. 1994. Elementary Mechanics of Soil Behaviour. Rotterdam: AA. Balkema.

Bishop AW. 1971. Shear strength parameters for undistributed and remolded soil specimens. In: Proceedings of the Roscoe Memorial Symposium on Stress Strain Behavior of Soils, Cambridge, pp. 3-58.

Bishop AW, Webb DL, Lewin PI. 1965. Undisturbed samples of London clay from Ashford Common shaft: strength - effective stress relationship. Géotechnique 15(1): 1-31.

Boudlal O, Khattaoui M, Kichi H. 2014. Caractérisation des marnes de la Grande Kabylie (Algérie). In: Actes des Journées Nationales de Géotechnique et de Géologie de l'Ingénieur JNGG2014, Beauvais.

Burland JB. 1990. On the compressibility and shear strength of natural soils. Géotechnique 40(3): 329-378.

Burland JB, Rampello S, Georgiannou VN, Calabresi G. 1996. A laboratory study of the strength of four stiff clays. Géotechnique 46 (3): 491-514

Cafaro F, Cotecchia F. 2001. Structure degradation and changes in the mechanical behaviour of a stiff clay due to weathering. Géotechnique 51(5): 441-453.

Callisto L, Rampello S. 2004. An interpretation of structural degradation for three natural clays. Can Geotech J 4(3): 392-407.

Chandler RJ. 1969. The effect of weathering on the shear strength properties of Keuper marl. Géotechnique 19(3): 321-334.

Chandler RJ. 1972. Lias clay: weathering processes and their effect on shear strength. Géotechnique 22(4): 403-431.

Chandler RJ, Apted JP. 1988. The effect of weathering on the strength of London Clay. Q J Eng Geol Hydrogeol 21: 59-68.

Clayton CRI, Serratrice JF. 1993. The mechanical properties and behaviour of hard soils and soft rocks. Rapport général de la session 2. In: Proceedings of the International Symposium on Geotechnical Engineering of Hard Soils and Soft Rocks, Athens, Vol. 3, pp. $1839-1877$.
Collins K, Mc Gown A. 1974. The form and function of microfabric features in a variety of natural soils. Géotechnique 24(2): 223-254.

Costa Filho LM. 1984. Technical note: a note on the influence of fissures on the deformation characteristics of London Clay. Géotechnique 34(2): 268-272.

Cotecchia F, Chandler RJ. 1997. The influence of structure on the pre-failure behaviour of a natural clay. Géotechnique 47(3): 523 544.

Cotecchia F, Chandler RJ. 2000. A general framework for the mechanical behaviour of clays. Géotechnique 50(4): 431-447.

Cotecchia F, Cafaro F, Aresta B. 2007. Structure and mechanical response of sub-Apennine Blue Clays in relation to their geological and recent loading history. Géotechnique 57(2): 167-180.

Croce A, Japelli R, Pellegrino A, Viggiani C. 1969. Compressibility and strength of stiff intact clays. In: Proceedings of 7 th International Conference on Soil Mechanics and Foundation Engineering, Mexico, Vol. 1, pp. 81-89.

Crooks JHA, Graham J. 1976. Geotechnical properties of the Belfast estuarine deposits. Géotechnique 26(2): 293-315.

Dehandschutter B, Vandycke S, Sintubin M, Vandenberghe N, Wouters L. 2005. Brittle fractures and ductile shear bands in argillaceous sediments: inferences from Oligocene Boom Clay (Belgium). J Struct Geol 27(6): 1095-1112.

Delage P, Lefebvre G. 1984. Study of the structure of a sensitive Champlain clay and its evolution during consolidation. Can Geotech J 21(1): 21-35.

Derriche Z, Cheikh-Lounis G. 2004. Caractéristiques géotechniques des marnes plaisanciennes d'Alger. Bull Eng Geol Environ 63: 367-378.

Djedid A, Bekkouche A, Aissa Mammoune SM. 2001. Identification et prévision du gonflement de quelques sols de la région de Tlemcen (Algerie). Bull Lab Ponts Chaussée 233: 67-75.

Donath F. Strength variation and deformational behavior in transversely isotropic rock. In : Judd WR, ed. State of stress in the earth's crust. New York: Elsevier, 1964, pp. 281-298.

Ejjaaouani H, Shakhirev V, Magnan JP, Bensallam S. 2013. Comportement des sols gonflants lors de l'humidification et du séchage. In: Proceedings of the 18th International Conference on Soil Mechanics and Geotechnical Engineering, Paris, pp. 1101-1104.

El Amrani N, Chacon J. 1996. Altérabilité, gonflement et propriétés d'indices des marnes néogènes de la dépression de Grenade (sud de l'Espagne). Bull Int Assoc Eng Geol 54: 77-89.

Fabre G. 2005. Fluage et endommagement des roches argileuses : évolution de la microstructure et modélisation phénoménologique. Thèse de Doctorat en Sciences, Université Joseph Fourier, Grenoble, 330 p.

Fabre G, Pellet F. 2006. Creep and time-dependent damage in argillaceous rocks. Int J Rock Mech Min Sci 43: 950-960.

Gasparre A, Nishimura S, Coop MR, Jardine RJ. 2007. The influence of structure on the behaviour of London clay. Géotechnique 57(1): 19-31.

Gasparre A, Nishimura S, Minh NA, Coop MR, Jardine RJ. 2007. The stiffness of natural London Clay. Géotechnique 57(1): 33-47.

Gasparre A, Coop MR. 2008. Quantification of the effects of structure on the compression of a stiff clay. Can Geotech J 45(9): 1324-1334.

Gaudin D. 1972. Pressions de terrain sur une galerie creusée dans des marnes du Stampien. Bull Liaison Ponts Chaussées 59: 133-155.

Gaudin B, Serratrice JF. 1998. Étude en laboratoire du comportement mécanique d'une molasse. In: Proceedings of the 2nd International Symposium on Hard Soils and Soft Rocks, Naples, pp. 173-181. 
Gaudin B, Folacci JP, Panet M, Salva L. 1981. Soutènement d'une galerie dans des marnes noires du Cénomanien. In: Proceedings of the 10th International Conference on Soil Mechanics and Foundation Engineering, Stockholm, Vol. 1/4, pp. 293-296.

Graham J, Noonan ML, Lew KV. 1983. Yield states and stress-strain relationships in a natural plastic clay. Can Geotech J 20(3): 502-516.

Graham J, Houlsby CTH. 1983. Anisotropic elasticity of a natural clay. Géotechnique 33(2): 165-180.

Guilloux A, Cojean R, Doré M, et al. 2005. Note sur la définition des «Sols Indurés Roches Tendres » (SIRT). Rev Fr Géotech 111: 59-66.

Hight DW, McMillan F, Powell JJM, Jardine RJ, Allenou CP. 2002. Some characterictics of London Clay. In: Proceedings of Symposium on Characterisation and Engineering Properties of Natural Soils, Singapore, Vol. 2, pp. 851-908.

Hoek E, Brown ET. 1980. Empirical strength criterion for rock masses. J Geotech Eng-ASCE 106(9): 1013-1035.

Houlsby GT, Wroth CP. 1991. The variation of the shear modulus of a clay with pressure and overconsolidation ratio. Soils Found 31(3): 138-143.

Jaeger JC. 1960. Shear failure of transversely isotropic rock. Geol Mag 97: 65-72.

Jamiolkowski M, Lancellotta R, Lo Presti D. 1995. Remarks on the stiffness at small strains of six Italian clays. In: Proceedings of the 1st International Symposium Pre-failure Deformation Characteristics of Geomaterials, IS-Hokkaido, Sapporo, pp. 817-836.

Kavvadas M, Anagnostopoulos A, Kalteziotis N. 1993. A framework for the mechanical behaviour of the Corinth marl. In: Proceedings of the International Conference on Hard Soils - Soft Rocks, Athens, Vol. 1, pp. 577-584.

Lamas F, Oteo C, Chacóna J. 2011. Influence of carbonate content on the stress-strength behaviour of neogene marls from the betic cordillera (Spain) in $\mathrm{cu}$ triaxial tests using a quasilinear elastic (hyperbolic) model. Eng Geol 122(3-4): 160-168.

Lee YL, Arafati N, Leca E, Magnan JP, Mestat P, Serratrice JF. 1993. Comportement et modélisation des marnes de Las Planas. In: Proceedings of the International Symposium on Geotechnical Engineering of Hard Soils and Soft Rocks, Athens, Vol. 2, pp. 1479-1486.

Leroueil S, Vaughan PR. 1990. The general and congruent effects of structure in natural soils and weak rocks. Géotechnique 40(3): 467-488.

Le Roux A. 1971. Le microscope électronique à balayage: application à l'étude des marnes. Bull Lab Ponts Chaussée 54: 6-12.

Le Roux A. 1972. Caractéristiques mécaniques des roches argileuses en relation avec leur texture. Bull Lab Ponts Chaussée 61: 155178.

Liao JJ, Yang MT, Hsieh HY. 1997. Direct tensile behavior of a transversely isotropic rock. Int J Rock Mech Min Sci 34(5): 831-849.

Lings ML, Pennigton DS, Nash DF. 2000. Anisotropic stiffness parameters and their measurement in a stiff natural clay. Géotechnique 50(2): 109-125.

Liu X, Buzzi O, Yuan S, Mendes J, Fityus S. 2016. Multi-scale characterization of retention and shrinkage behaviour of four Australian clayey soils. Can Geotech J 53(5): 854-870.

Magnan JP, Serratrice JF. 1995. Détermination de la courbe d'état limite d'une marne. In: Proceedings on the 11th European Conference on Soil Mechanics and Foundation Engineering, Copenhague, Vol. 3, pp. 1839-1847.

Maquaire O, Malet JP, Remaître A, Locat J, Klotz S, Guillon J. 2003. Instability conditions of marly hillslopes: towards landsliding or gullying? The case of the Barcelonnette Basin, South East France. Eng Geol 70: 109-130.
Marsland A. 1971. The shear strength of stiff fissured clays. Stress-strains behaviour of soils. In: Proceedings of the Roscoe Memorial Symposium on Stress Strain Behavior of Soils, pp. 59-68.

Mitchell JK. 1976. Fundamentals of soil behavior. New York: Wiley.

Mouhssine M, Touzani A, Zinoune S, Mahtal A. 2015. Terrassements sur terrains marneux sensibles aux glissements : cas des terrains et remblais autoroutiers entre Oued Amlil et Taza (Rif, Maroc). Physio-Géo 9(1): 61-80.

Nasseri MH, Rao K, Ramamurthy T. 2003. Transversely isotropic strength and deformational behavior of Himalayan schists. Int $J$ Rock Mech Min Sci 40(1): 3-23.

Pejon OJ, Le Roux A, Guignard D. 1997. Comportement à l'eau des roches argilo-marneuses, suivi du gonflement, importance de laminéralogie et des textures. Bull Assoc Int Géol Ing 55: 105-119.

Picarelli L, Urciuoli G, Mandolini A, Ramondini M. 2006. Softening and instability of natural slopes in highly fissured plastic clay shales. Nat Hazards Earth Syst Sci 6: 529-539.

Raynaud S, Ngan-Tillard D, Desrues J, Mazerolle F. 2008. Brittle-toductile transition in Beaucaire marl from triaxial tests under the CT-scanner. Int J Rock Mech Min Sci 45(5): 653-671.

Serrano AA, Oteo C, Dapena E, Vinas JM. 1981. Analysis of swelling phenomena in a gypsum-marl formation. In: Proceedings of the 10th International Conference Soil Mechanics and Foundation Engineering, Stockholm, Vol. 1/4, pp. 779-784.

Serratrice JF, 1995. Essais de laboratoire à haute pression sur une marne. In: Actes du Colloquium Mundanum Craies et Schistes, Bruxelles, pp. 61-70.

Serratrice JF. 1998. Caractéristiques mécaniques des marnes de l'Oligocène du bassin de Marseille. In: Proceedings of the 2nd International Symposium on Hard Soils and Soft Rocks, Naples, pp. 301-310.

Serratrice JF. 2002. Outils et procédures de caractérisation des sols indurés et des roches tendres : l'expérience du LRPC d'Aix-enProvence. In: Actes du Symposium International PARAM 2002, Paris, pp. 313-326.

Serratrice JF. 2011. Propriétés mécaniques d'une marne. In: Proceedings on the 15th European Conference on Soil Mechanics and Geotechnical Engineering, Athènes, pp. 459-464.

Serratrice JF. 2013. Essais triaxiaux sur des marnes fissurées. In: Actes des $3^{\text {ème }}$ Conférence Maghrébine en Ingénierie Géotechnique GMIG'13, Alger, pp. 345-350.

Serratrice JF, Calissano H, Batilliot L. 2012. Résistances triaxiales d'une marne et de son argile d'altération. In: Actes des Journées Nationales de Géotechnique et de Géologie de l'ingénieur JNGG 2012, Bordeaux, pp. 427-434.

Sivakumar V, Doran IG, Graham J, Johnson A. 2001. The effect of anisotropic elasticity on the yielding characteristics of overconsolidated natural clay. Can Geotech J 38(1): 125-137.

Skempton AW. 1970. First-time slides in overconsolidated clays. Géotechnique 20(3): 320-324.

Skempton AW, Petley DJ. 1967. The strength along structural discontinuities in stiff clays. In: Proceedings of the Geotechnical Conference, Oslo, Vol. 2, pp. 29-46.

Skempton AW, Schuster FRS, Petley DJ. 1969. Joints and fissures in the London clay at Wraysbury and Edgware. Géotechnique 19(2): 205-217.

Tavenas F, Leroueil S. 1977. Effects of stresses and time on yielding of clays. In: Proceedings of the 9th International Conference on Soil Mechanics and Foundation Engineering, Tokyo, Vol. 1, pp. 319-326. 
Tavenas F, Leroueil F. 1979. Les concepts d'état limite et d'état critique et leurs applications pratiques à l'étude des argiles. Rev Fr Géotech 6: 27-49.

Tillard-Ngan D, Desrues J, Raynaud S, Mazerolle F. 1993. Strain localisation in Beaucaire marl. In: Proceedings of the International Symposium on Geotechnical Engineering of Hard Soils and Soft Rocks, Athens, Vol. 2, pp. 679-1686.

Tran TD. 2014. Rôle de la microstructure des sols argileux dans les processus de retrait-gonflement : de l'échelle de l'éprouvette à l'échelle de la chambre environnementale. Thèse de Doctorat, école Nationale Supérieure des Mines de Paris, ParisTech, $216 \mathrm{p}$.

Vitone C, Cotecchia F. 2011. The influence of intense fissuring on the mechanical behaviour of clays. Géotechnique 61(12): 1003-1018.

Yang C, Carter JP, Sheng D. 2014. Description of compression behaviour of structured soils and its application. Can Geotech $J$ 51(8): 921-933.

Citation de l'article : Jean François Serratrice. Divers aspects du comportement mécanique des marnes en laboratoire. Rev. Fr. Geotech. $2017,151,3$. 